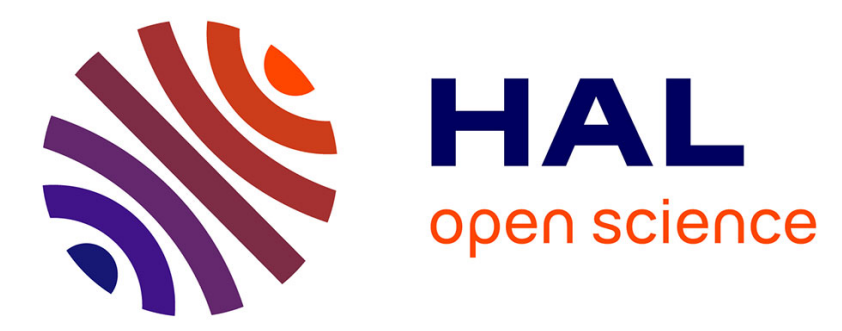

\title{
The fluctuation-dissipation theorem as a diagnosis and cure for zero-point energy leakage in quantum thermal bath simulations
}

Etienne Mangaud, Simon Huppert, Thomas Plé, Philippe Depondt, Sara Bonella, Fabio Finocchi

\section{To cite this version:}

Etienne Mangaud, Simon Huppert, Thomas Plé, Philippe Depondt, Sara Bonella, et al.. The fluctuation-dissipation theorem as a diagnosis and cure for zero-point energy leakage in quantum thermal bath simulations. Journal of Chemical Theory and Computation, 2019, 15 (5), pp.2863-2880. 10.1021/acs.jctc.8b01164 . hal-02171352

\section{HAL Id: hal-02171352 \\ https://hal.sorbonne-universite.fr/hal-02171352}

Submitted on 2 Jul 2019

HAL is a multi-disciplinary open access archive for the deposit and dissemination of scientific research documents, whether they are published or not. The documents may come from teaching and research institutions in France or abroad, or from public or private research centers.
L'archive ouverte pluridisciplinaire HAL, est destinée au dépôt et à la diffusion de documents scientifiques de niveau recherche, publiés ou non, émanant des établissements d'enseignement et de recherche français ou étrangers, des laboratoires publics ou privés. 


\title{
The fluctuation-dissipation theorem as a diagnosis and cure for zero-point energy leakage in quantum thermal bath simulations
}

\author{
Etienne Mangaud, ${ }^{1}$ Simon Huppert, ${ }^{1}$ Thomas Plé, ${ }^{1}$ Philippe Depondt, ${ }^{1}$ Sara Bonella, ${ }^{2}$ and Fabio Finocchi ${ }^{1}$ \\ 1) Sorbonne Université, CNRS, Institut des NanoSciences de Paris, INSP, 4 place Jussieu, F-75005 Paris, \\ France \\ ${ }^{2)}$ CECAM Centre Européen de Calcul Atomique et Moléculaire, École Polytechnique Fédérale de Lausanne, \\ Batochimie, Avenue Forel 2, 1015 Lausanne, Switzerland
}

\begin{abstract}
Quantum thermal bath (QTB) simulations reproduce statistical nuclear quantum effects via a Langevin equation with a colored random force. Although this approach has proven efficient for a variety of chemical and condensed-matter problems, the QTB, as many other semiclassical methods, suffers from zero-point energy leakage (ZPEL). The absence of a reliable criterion to quantify the ZPEL without resorting to demanding comparisons with path integral based calculations has so far hindered the use of the QTB for the simulation of real systems. In this work, we establish a quantitative connection between ZPEL in the QTB framework and deviations from the quantum fluctuation-dissipation theorem (FDT) that can be monitored along the simulation. This provides a rigorous general criterion to detect and quantify the ZPEL without any a priori knowledge of the system under study. We then use this criterion to build an adaptive QTB method that strictly enforces the quantum FDT at all frequencies via an on-the-fly, spectrally resolved fine tuning of the system-bath coupling coefficients. The validity of the adaptive approach is first demonstrated on a simple two-oscillator model. It is then applied to two more realistic problems: the description of the vibrational properties of a model aluminium crystal at low temperature and the simulation of the liquid-solid phase transition in a 13-atom neon cluster. In both systems, the standard QTB results are strongly altered by the ZPEL, which can be essentially eliminated using the adaptive approach.
\end{abstract}

\section{INTRODUCTION}

Accounting for nuclear quantum effects in molecular dynamics (MD) simulations of complex atomic systems is an important issue when light elements, such as hydrogen, are involved, or for the description of lowtemperature processes. However, modelling these effects remains a theoretical challenge for which several different approaches have been proposed. For the static properties of molecular and condensed matter systems, the reference methods are based on Feynman's path integral formalism ${ }^{1-4}$. Average values of quantum operators are obtained exactly by performing MD or Monte Carlo simulations on a sufficient number, $P$, of replicas of the system under study. These methods can be particularly resource-consuming when the value of $P$ required to converge to the exact result becomes large, e.g. at low temperatures. The simulation of dynamical properties such as time correlation functions is even more challenging and all of the simulation techniques applicable to complex multi-atomic systems imply strong approximations that are difficult to control. Notable examples of these methods are the extensions of the path integral MD that provide approximate time correlation functions, namely ring-polymer $\mathrm{MD}^{5,6}$ and centroid $\mathrm{MD}^{7,8}$, as well as the various semiclassical methods in which dynamical properties are calculated by averaging over classical trajectories with initial conditions sampled according to the quantum distribution ${ }^{9-14}$.

In the past decade new approaches have also been developed in which the quantum delocalization of the nuclei is introduced approximately using a Langevin equation with a colored noise. The quantum thermostat ${ }^{15,16}$ and the quantum thermal bath ${ }^{17}$ (QTB) methods, although different in their formal expressions, both rely on the same principle: in the colored-noise Langevin dynamics, each vibrational mode of the system is thermalized, not at the physical temperature $T$, but at an effective temperature that includes the appropriate zero-point energy, so that the stochastic dynamics approximately reproduces the zero-point motion. Both variants of the quantumcolored Langevin equation have been shown to provide good qualitative and quantitative results in various practical cases ${ }^{18-23}$ with only a small numerical overhead with respect to classical MD and therefore at a much lower computational expense than path integral MD. In particular, some studies have shown the vibrational spectra obtained within the QTB to agree with experimental data or with the results of more costly quantum simulation methods even when strong nuclear quantum effects were involved ${ }^{24-26}$. As in any Langevin dynamics, the system-bath coupling induces a broadening and possibly deformations of the vibrational spectrum, which limit the use of the QTB for the simulation of dynamical properties. Recently developed techniques ${ }^{23,27}$, however, might allow to deconvolute the spectra from the effect of the bath and to recover the unaffected dynamics.

In spite of the advantages described above, and as many other semiclassical approaches, the colored Langevin methods suffer from a major flaw: the zero point energy leakage ${ }^{28-33}$ (ZPEL, see description in section II).

As a possible solution to this issue, colored-noise path integral MD approaches have been developed in which the number $P$ of replicas can be reduced with respect to standard path integral MD without inducing significant $\mathrm{ZPEL}^{34,35}$. Although $P$ is reduced, it can still be large in 
the case of low-temperature simulations ${ }^{36}$. Furthermore, to our knowledge, this approach has not been adapted to compute dynamical properties so far. In the context of semiclassical methods, a recent work ${ }^{37}$ has shown that one particular approach, the Herman-Kluk propagator, is free from ZPEL. Although this demonstration is encouraging for further developments, the numerical cost of this accurate simulation technique remains high and can be problematic for applications to large systems. Within the framework of the QTB, it was shown that increasing the system-bath coupling reduces the ZPEL substantially ${ }^{32}$. This approach, however, affects the dynamical and even the static properties of the simulated system and can hinder the accuracy of the QTB results. A clear criterion to quantify the ZPEL is thus needed to enable more reliable and general applications of the QTB.

Existing studies ${ }^{31-33}$ rely either on comparisons with path integral results or on normal-mode decompositions to assess the presence of ZPEL in QTB simulations and the quality of the quantum distribution sampling. Normal mode analysis, however, is valid only for weakly anharmonic systems, while comparisons with path integrals reintroduce high numerical costs. In this work, we propose an alternative approach that circumvents both problems. We show, in the framework of the linear response theory, that the quantum fluctuation-dissipation theorem (FDT) provides, at a very low computational cost, a self-consistent assessment of the ZPEL in QTB simulations, without resorting to normal-mode or path integral calculations. The quantification of the ZPEL can then be used to modify the system-bath coupling in order to compensate for the leakage and restore the quantum FDT. This leads to a new QTB algorithm, the adaptive QTB (adQTB) that extends significantly the capabilities of this approach with limited increase in the numerical cost.

The article is organized as follows: in section II, we discuss the issue of the ZPEL in QTB simulations. In section III, we introduce the quantum fluctuationdissipation relation that enables a diagnosis of ZPEL in QTB simulations and illustrate it with a simple twooscillator model. In section IV, we use the fluctuationdissipation relation to define two adaptive variants of the QTB in which the ZPEL is compensated via an on-thefly spectrally resolved tuning of the system-bath coupling coefficients. Finally, in section V, the adaptive QTB schemes are applied to two more realistic problems that have, so far, presented serious challenges for the QTB. We study first a Lennard-Jones aluminium crystal at low temperatures and show that, in contrast with the standard implementation of $\mathrm{QTB}^{31,32}$, the energy distribution amongst the normal modes is correctly captured by the adaptive schemes. We then discuss QTB simulation of a 13-atom neon cluster. In this strongly anharmonic system, ZPEL causes the standard algorithm to predict an abnormal liquid-like phase at low temperatures ${ }^{33}$. We analyze the conditions under which this failure of the QTB can be corrected by the adaptive scheme.
Our focus in this paper is on presenting the new FDT criterion and demonstrating its potential on a first set of recognized benchmark systems. The numerical results of the adaptive QTB are very encouraging, but further tests will be necessary to fully assess the capabilities of the method and its numerical efficiency with respect to alternative approaches (e.g. path integral MD). To convey the key theoretical points more effectively and illustrate more clearly the performances of the proposed algorithms, we omit some technical details on their practical implementation from the main text. This information is, however, detailed in the Appendices.

\section{QTB AND ZERO-POINT ENERGY LEAKAGE}

In the quantum thermal bath method, each nuclear degree of freedom follows a Langevin equation of the type:

$$
m_{k} \dot{v}_{k}=-\frac{\partial V}{\partial x_{k}}-m_{k} \gamma v_{k}+F_{k}(t)
$$

In this expression, $v_{k}$ and $m_{k}$ are the velocity and the mass of the degree of freedom $k, V$ is the interaction potential and the $F_{k}(t)$ are Gaussian random forces, with cross-correlation spectra (defined as the Fourier transform of the time correlation functions) given by:

$$
C_{F_{k} F_{j}}(\omega)=2 m_{k} \gamma \theta(\omega, T) \delta_{k}^{j}
$$

where $\delta_{k}^{j}$ denotes the Kronecker symbol and

$$
\theta(\omega, T)=\hbar \omega\left[\frac{1}{2}+\frac{1}{\mathrm{e}^{\frac{\hbar \omega}{k_{B} T}}-1}\right]
$$

In eq. (2), the thermal energy $k_{B} T$ of the classical Langevin equation is replaced by the energy $\theta(\omega, T)$ of a quantum harmonic oscillator at frequency $\omega$ and temperature $T$. The strength of the system-bath coupling is characterized by $\gamma$, which appears both as a damping coefficient in eq. (1) and in the amplitude of the Langevin random forces in eq. (2).

In the QTB method, the interaction of the system with the quantum bath (via the random and friction forces) tends to thermalize each vibrational mode at the effective thermal energy $\theta(\omega, T)$ that increases with the mode frequency $\omega$ and accounts for for zero-point energy. [...] However, in the presence of anharmonicity, the classical forces $-\frac{\partial V}{\partial x_{k}}$ couple the different modes and tend to drive the system towards the classical equipartition of energy. As a consequence, the average thermal energy per mode effectively obtained through the QTB is in general less than $\theta(\omega, T)$ for large $\omega$ and greater than it for small $\omega$. This unphysical energy flow from high to low frequencies constitutes the zero-point energy leakage. This leakage can have dramatic consequences, such as the spurious melting of solids (see section VB).

In Ref. 32, the use of large system-bath coupling constants $\gamma$ was proposed to effectively enforce the quantum 
energy distribution $\theta(\omega, T)$ and limit the effect of the ZPEL. This strategy proved efficient for mildly anharmonic systems ${ }^{32}$, but it has two serious limitations that we both address in the present paper.

Firstly, although the impact of the ZPEL is reduced for large $\gamma$, the leakage is not fully eliminated ${ }^{32}$. As mentioned in the introduction, a general criterion is still lacking to estimate the importance of the ZPEL and define an acceptable level for the associated error. In section III, we propose such a general criterion, based on the quantum FDT, that allows to quantify the ZPEL using correlation spectra computed along the QTB trajectory.

Secondly, in the QTB, $\gamma$ cannot be increased arbitrarily without affecting the accuracy of the results. Indeed, the friction force is responsible for a spectral broadening of the vibrational modes that may affect the quality of the simulated spectra and even cause a bias on the estimates for static properties ${ }^{38,39}$ (this point is examined in more detail in appendix C). As a general rule of thumb, $\gamma$ should be kept small compared to the frequencies of the quantum modes of the system, i.e. the modes for which the zero-point energy is relevant. However, this condition is problematic for the simulation of highly anharmonic systems, for which a large $\gamma$ is needed to control the ZPEL. The adQTB method proposed in section IV makes it possible to suppress the leakage using values of $\gamma$ much smaller than the ones needed in standard QTB, therefore improving the accuracy of the QTB approach and extending its domain of applicability.

\section{FLUCTUATION-DISSIPATION CRITERION FOR THE ZPEL}

In this section we show that an appropriately adapted version of the quantum fluctuation-dissipation theorem (FDT) enables to detect and quantify the ZPEL in QTB simulations. To set the stage, we start by rewriting the position-velocity FDT in a more convenient form involving the velocity power spectrum and argue why this is a relevant starting point for our considerations. Then, in section III B, we show how to express the linear susceptibility - in general not accessible analytically or numerically - in terms of quantities that can be computed along a QTB trajectory. This expression for the susceptibility will make it possible to monitor discrepancies from the FDT and gauge the ZPEL based directly on results from the QTB simulation, thus providing us with a general diagnosis tool applicable to realistic systems. We close this section by illustrating the FDT criterion on a simple two-oscillator model considered in Ref. 32.

\section{A. The FDT as a diagnosis tool for ZPEL}

Let us start by recalling the fluctuation-dissipation theorem, a fundamental law of linear response theory, given for the relevant case of position and velocity operators as (see Ref. 40):

$$
\operatorname{Re}\left[\chi_{v x}(\omega)\right]=\frac{\omega}{2 i \theta(\omega, T)} \int_{-\infty}^{+\infty}\langle\{x(0) v(t)\}\rangle \mathrm{e}^{-i \omega t} d t,
$$

where $\theta(\omega, T)$ is the quantum thermal energy in equation (3), $\chi_{x v}(\omega)$ is the linear susceptibility and $\langle\{x(0) v(t)\}\rangle=$ $\frac{1}{2} \operatorname{Tr}\left[(x(0) v(t)+v(t) x(0)) \rho_{e q}\right]$ denotes the symmetrized average of the position and velocity operators over the quantum equilibrium probability density $\rho_{e q}$. Using the time translation invariance property $\langle\{x(0) v(t)\}\rangle=$ $\langle\{x(-t) v(0)\}\rangle$ and an integration by parts, the FDT can be rewritten as a function of the velocity autocorrelation spectrum:

$$
\operatorname{Re}\left[\chi_{v x}(\omega)\right]=\frac{C_{v v}(\omega)}{2 \theta(\omega, T)}
$$

Eq. (5), is an exact result that reflects the quantum distribution of the thermal energy, characterized by the function $\theta(\omega, T)$. It is satisfied at each frequency if the quantum operators $x$ and $v$ evolve according to the exact quantum dynamics. In the QTB dynamics, however, operators are replaced by classical variables, and the colored thermostat used to induce the energy distribution $\theta(\omega, T)$ is combined with a purely classical, deterministic evolution of positions and velocities. As a consequence, the resulting stochastic dynamics violates the quantum FDT for anharmonic systems. In particular, the drive towards equipartition of energy introduced by the classical evolution originates the ZPEL. Monitoring the violation of eq. (5) along a QTB trajectory thus provides a general criterion to detect and quantify the ZPEL in a simulation.

In practice, to apply this criterion, all quantities appearing in eq. (5) must be evaluated along the QTB trajectory. In this framework, statistical averages over quantum operators are replaced by their classical counterparts. The Fourier transform of the velocity autocorrelation function $C_{v v}(\omega)$ can then be straightforwardly computed. The evaluation of the susceptibility $\chi_{x v}(\omega)$, on the other hand, is non trivial. In the next section, we show that, in the QTB framework, the dissipative part of the susceptibility $\operatorname{Re}\left[\chi_{x v}(\omega)\right]$ can be expressed as the ratio of two correlation functions that can be evaluated directly.

\section{B. Linear response theory and correlation functions}

The susceptibility $\chi_{v x}(\omega)$ characterizes the linear response of the system at thermal equilibrium, subject to a small perturbative force $\Delta F(t)$. The linear velocity response to $\Delta F$ is given in the frequency domain by the following relation ${ }^{40}$ :

$$
\Delta v(\omega)=\chi_{v x}(\omega) \Delta F(\omega)
$$

In general, the susceptibility cannot be computed analytically but it can be estimated numerically in a QTB 
simulation, by considering the linear response of the system to the stochastic Langevin force. More precisely, relation (6) cannot be applied directly since the Langevin force $F(t)$ cannot be treated as a small perturbation. To circumvent this difficulty, we consider the results that would be obtained in two different simulations, one with the QTB-Langevin force $F(t)$, and an other (virtual) one with the modified force $F^{\prime}(t)$ which is defined from $F(t)$ by setting to zero all its frequency-components in the vicinity of a particular angular frequency $\omega_{0}$. The force $F^{\prime}$ is expressed in the frequency domain as:

$$
F^{\prime}(\omega)=F(\omega)\left[1-\Pi\left(\omega-\omega_{0}\right)\right]
$$

where the rectangular function $\Pi\left(\omega-\omega_{0}\right)$ equals 1 inside the interval $\left[\omega_{0}-\frac{\Delta \omega}{2}, \omega_{0}+\frac{\Delta \omega}{2}\right]$ and zero outside. Using the definition above, the force difference between the original and the modified QTB dynamics, $\Delta F(t)=$ $F(t)-F^{\prime}(t)$, is given by:

$$
\Delta F(t)=\int_{\omega_{0}-\frac{\Delta \omega}{2}}^{\omega_{0}+\frac{\Delta \omega}{2}} \frac{d \omega}{\pi} \operatorname{Re}\left[F(\omega) \mathrm{e}^{i \omega t}\right]
$$

If $\Delta \omega$ is small enough, the force $\Delta F(t)$ can be treated as a small perturbation. In practice, in QTB simulations, the trajectory is finite, the force spectrum is discrete and $\Delta \omega=\frac{2 \pi}{\tau}$, where $\tau$ is the total duration over which the correlation spectra are evaluated (see appendix B for details). Relation (6) can then be applied to express the linear velocity response $\Delta v(\omega)$ as a function of the perturbative force spectrum $\Delta F(\omega)$. To that end, we multiply both members of equation (6) by $\Delta F(-\omega)$ and use the relation between the cross-correlation spectrum and the Fourier transform, $C_{A B}(\omega) \propto\langle A(\omega) B(-\omega)\rangle$ - here the symbol $\langle\ldots\rangle$ refers to the average value for the stochastic QTB dynamics. This yields:

$$
\operatorname{Re}\left[C_{\Delta v \Delta F}(\omega)\right]=\operatorname{Re}\left[\chi_{v x}(\omega)\right] C_{\Delta F \Delta F}(\omega),
$$

We then notice that:

$$
C_{\Delta v \Delta F}(\omega)=C_{v \Delta F}(\omega)-C_{v^{\prime} \Delta F}(\omega)
$$

This relation can be simplified by noting that $C_{v^{\prime} \Delta F}(\omega) \propto\left\langle v^{\prime}(\omega) \Delta F(-\omega)\right\rangle=0$. To prove this statement, we make use of the fact that the different frequency-components of $F$ are uncorrelated random variables: $\left\langle F(\omega) F\left(\omega^{\prime}\right)\right\rangle \propto \delta\left(\omega+\omega^{\prime}\right)$. This property is verified by construction by the colored Langevin force and it is valid for any system (see appendix A for details on the generation of the random force). It implies that $v^{\prime}$, which is generated by the random force $F^{\prime}$, is statistically independent of $\Delta F$, so that:

$$
\left\langle v^{\prime}(\omega) \Delta F(-\omega)\right\rangle=\left\langle v^{\prime}(\omega)\right\rangle\langle\Delta F(-\omega)\rangle=0,
$$

where we have used the fact that the frequency components of $F$ have zero mean value. Equation (9) is then rewritten as:

$$
\operatorname{Re}\left[C_{v \Delta F}(\omega)\right]=\operatorname{Re}\left[\chi_{v x}(\omega)\right] C_{\Delta F \Delta F}(\omega)
$$

Furthermore, for $\omega$ in the interval $\left[\omega_{0}-\frac{\Delta \omega}{2}, \omega_{0}+\frac{\Delta \omega}{2}\right]$, $\Delta F(\omega)=F(\omega)$, therefore the following relations hold:

$$
C_{v \Delta F}(\omega)=C_{v F}(\omega) \text { and } C_{\Delta F \Delta F}(\omega)=C_{F F}(\omega)
$$

Substituting the identities above in eq. (11), we finally obtain an expression for the dissipative part of the susceptibility $\operatorname{Re}\left[\chi_{v x}(\omega)\right]$ in terms of the correlation functions $C_{F F}(\omega)$, that is known, and $C_{v F}(\omega)$, that can be computed along a QTB trajectory. This expression is given by:

$$
\operatorname{Re}\left[\chi_{v x}(\omega)\right]=\frac{\operatorname{Re}\left[C_{v F}(\omega)\right]}{C_{F F}(\omega)}
$$

The proof derived above only applies for $\omega$ close to $\omega_{0}$, but since no particular assumption was made on $\omega_{0}$, the relation (12) holds for any value of $\omega$.

It might seem surprising at first sight that the random force is treated here as a perturbation in the determination of $\chi_{v x}$. Indeed, in Langevin simulations the random force is essential to drive the system to its equilibrium and it cannot be considered small. Nevertheless, what the argument reported above shows is that $C_{v F}(\omega)$ can be related to the linear susceptibility, without the need to consider possible contributions of the nonlinear response to the random force $F(t)$. It should be stressed that this is a statistical property that follows from the fact that the different frequency-components of $F$ are uncorrelated random variables with zero mean value. The nonlinear response to $F(t)$ can still be significant, and although the corresponding contributions average to zero when computing $C_{v F}(\omega)$, they increase the noise on its estimate from the QTB trajectory.

\section{ZPEL diagnosis}

We rewrite eq. (5) using eq. (12) and the expression for the random force autocorrelation function $C_{F F}(\omega)$ as given by eq. (2) within the QTB method. This yields:

$$
\operatorname{Re}\left[C_{v F}(\omega)\right]=m \gamma C_{v v}(\omega)
$$

As both sides of eq. (13) can be computed along QTB trajectories, this reformulation of the FDT can be applied directly to quantify the ZPEL. The terms in eq. (13) can also be given a direct physical interpretation: $\operatorname{Re}\left[C_{v F}(\omega)\right]$ corresponds to the spectrum of the power injected into the system by the random force, while the power dissipated by the friction force is given by $m \gamma C_{v v}(\omega)$.

From a computational point of view it is worth stressing that the fluctuation-dissipation theorem - from which equation (13) is derived in the framework of the QTB holds independently for each degree of freedom of a quantum system and its verification does not require a normal mode decomposition or any knowledge a priori of the system under study. 
Let us illustrate the use of eq. (13) on the model Hamiltonian introduced in Ref. 32:

$H=\frac{\dot{x}_{1}^{2}}{2}+\frac{\dot{x}_{2}^{2}}{2}+\omega_{1}^{2} \frac{x_{1}^{2}}{2}+\omega_{2}^{2} \frac{x_{2}^{2}}{2}+c_{3}\left(x_{1}-x_{2}\right)^{3}+c_{4}\left(x_{1}-x_{2}\right)^{4}$

It consists of two harmonic oscillators at frequencies $\omega_{1}$ and $\omega_{2}$ (the degrees of freedom $x_{1}$ and $x_{2}$ are dimensionless and the masses are equal to 1 ), coupled via anharmonic terms characterized by the two coefficients $c_{3}$ and $c_{4}$. In this system, the amount of ZPEL is controlled by the tunable parameters $c_{3}$ and $c_{4}$, and exact reference results can be easily obtained through the numerical solution of the associated Schroedinger equation. The simulations are performed at low temperature $T \ll \omega_{1}, \omega_{2}$ ( $k_{B}=\hbar=1$ in our system of units), so that the energy in each degree of freedom consists mainly of zero-point energy.
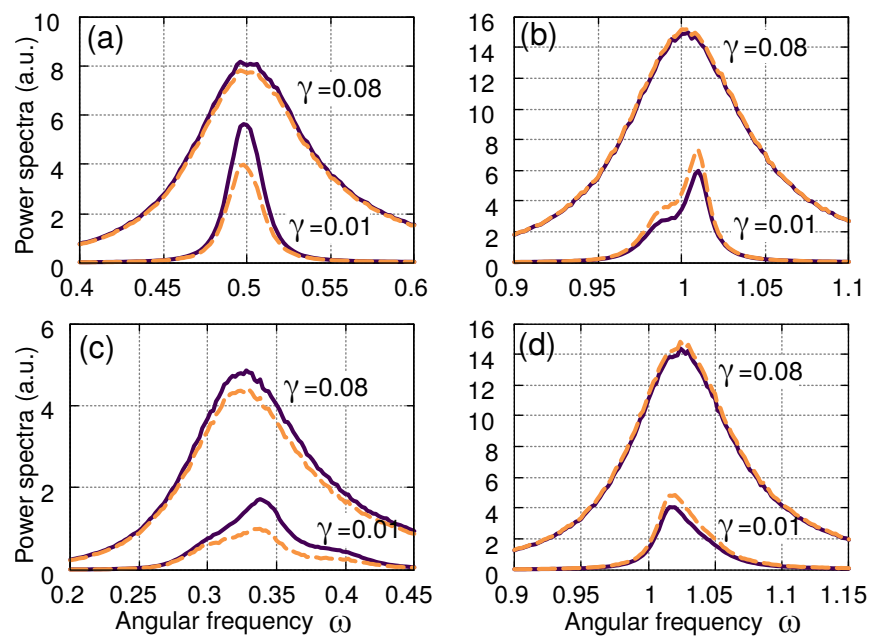

FIG. 1. Injected power spectrum $\operatorname{Re}\left[C_{v F}(\omega)\right]$ (dashed orange) and dissipated power spectrum $m \gamma C_{v v}(\omega)$ (dark magenta) for oscillator 1 (high frequency, panels a and c) and oscillator 2 (low frequency, panels $b$ and $d$ ) in the model of equation (14). The value of $\omega_{1}$ is fixed to 1 . In panel (a) and (b), $\omega_{2}=0.5, c_{4}=0$ and $c_{3}=3.8 \times 10^{-3}$. In panel (c) and (d), $\omega_{2}=0.25, c_{3}=0$ and $c_{4}=1.9 \times 10^{-3}$. These values are chosen in order to generate significant $\mathrm{ZPEL}^{32}$. We use natural units, $\hbar=1, k_{B}=1$ and $T=0.03 \ll \omega_{1}, \omega_{2}$. The timestep is $d t=0.063$ and the angular frequency cutoff ${ }^{38}$ is $\omega_{\text {cut }}=2$.

Each figure shows the curves obtained for $\gamma=0.01$ and

$$
\gamma=0.08 \text {. }
$$

In the harmonic case $\left(c_{3}=c_{4}=0\right.$, not shown $)$ the spectra on the right and left hand side of eq. (13) are equal, presenting Lorentzian peaks of width $\gamma$ and centered at the frequencies $\omega_{k}$ : in that case there is no ZPEL and the injected power equals the dissipated power. Each oscillator is then thermalized by the QTB with the correct thermal energy distribution $\theta\left(\omega_{k}, T\right)$ and the quantum FDT is satisfied. On the contrary, when anharmonicity is introduced, leakage occurs: for the highfrequency mode (Fig. 1.b and 1.d), the injected power spectrum lies above the dissipated power spectrum, while the reverse is true for the low-frequency mode (Fig. 1.a and 1.c).

The results are sensitive to the coupling constant $\gamma$. For $\gamma=0.01$, the difference between the injected and dissipated power spectra is large. This reflects a massive ZPEL that causes significant errors in the evaluation of the average energy in each mode, as examined in detail in section IV. Increasing $\gamma$ to 0.08 makes the ZPEL less important compared to the total power exchanged with the bath. Although the leakage is not completely suppressed, its impact is reduced and the average thermal energy in each mode approaches the correct quantum distribution $\theta\left(\omega_{k}, T\right)$, as it was noted in Ref. 32 .

\section{ADAPTIVE QUANTUM THERMAL BATH}

\section{A. Principles of the adaptive QTB}

In the previous section, we re-expressed the quantum fluctuation-dissipation theorem in a form suitable to detect and quantify the ZPEL in QTB simulations. Note that, since eq. (13) relates two spectra, it naturally provides an estimate of the leakage at each frequency. In this section, we build on this framework to present an adaptive QTB scheme in which the quantum FDT is actively enforced on the fly using frequency-dependent coefficients for the system-bath coupling. To that end, let us begin by introducing the following generalized version of the QTB Langevin equation:

$$
m \dot{v}=-\frac{\partial V}{\partial x}-m \int_{0}^{\infty} \tilde{\gamma}_{f}(\tau) v(t-\tau) d \tau+F(t)
$$

where $F(t)$ is now a Gaussian random force with an autocorrelation function given by:

$$
C_{F F}(\omega)=2 m \gamma_{r}(\omega) \theta(\omega, T) .
$$

In the generalized Langevin equation proposed above, two distinct system-bath coupling coefficients were introduced: the friction coefficient $\tilde{\gamma}_{f}$, and the random force coefficient $\gamma_{r}$. Furthermore, these coefficients were made time or frequency dependent. With these notations, the standard QTB method corresponds to the choice $\gamma_{r}(\omega)=\gamma_{f}(\omega)=\gamma$, with $\gamma_{f}(\omega)$ the Fourier transform of the friction kernel $\tilde{\gamma}_{f}(\tau)$. Taking into account the frequency dependence of $\gamma_{r}$ and following the arguments of section III, the fluctuation-dissipation theorem now takes the form:

$$
\operatorname{Re}\left[C_{v F}(\omega)\right]=m \gamma_{r}(\omega) C_{v v}(\omega)
$$

In the following, this equation will be referred to as the quantum FDT. It is worth stressing that, because we now distinguish between $\gamma_{r}$ and $\gamma_{f}$, equation (17) is not simply the expression of the equality between injected and dissipated power. Indeed, the dissipated power would be 
obtained by replacing $\gamma_{r}$ by $\gamma_{f}$ in the right-hand side of eq. (17). We also define the deviation from the FDT:

$$
\Delta_{F D T}(\omega)=\operatorname{Re}\left[C_{v F}(\omega)\right]-m \gamma_{r}(\omega) C_{v v}(\omega)
$$

In an adQTB simulation, $\Delta_{F D T}(\omega)$ is evaluated along the QTB dynamics and the memory kernels $\gamma_{r}(\omega)$ and $\gamma_{f}(\omega)$ are adapted in order to minimize this deviation and reach the correct average energy distribution for each frequency. The basic idea of this scheme is schematized in Fig. 2.c: in order to compensate for the leakage, the random force amplitude $\gamma_{r}(\omega)$ is increased at high frequencies and decreased at low frequencies, while the friction coefficient $\operatorname{Re}\left[\gamma_{f}(\omega)\right]$ is varied in the opposite direction. Importantly, these adjustments are performed on the fly, based on a self-tuning procedure that relies only on quantities computed from the QTB trajectory (a detailed description of this procedure is provided in appendix B). The quantum FDT (17) is independently valid for each degree of freedom of the system. Consistently, in the adQTB, specific coefficients $\gamma_{f}$ and $\gamma_{r}$ are associated to each degree of freedom and each pair of coefficients is adjusted separately to enforce the corresponding FDT.

In the following, we consider two variants of the method and compare their performances. In the first one, denoted adQTB-r, $\gamma_{r}(\omega)$ is adapted, while the friction kernel is kept at a fixed value $\gamma_{f}(\omega)=\gamma$, independently of $\omega$. In the second variant, denoted adQTB-f, $\gamma_{f}(\omega)$ is adjusted, while the random force kernel is kept at the value $\gamma_{r}(\omega)=\gamma$. In all the applications presented in this paper, at the beginning of the simulations both kernels are initialized at the value $\gamma_{r}(\omega)=\gamma_{f}(\omega)=\gamma$, then one of the two (depending on the adQTB variant) is dynamically adapted to enforce the FDT. However, the adaptive scheme might also be used with a different choice of initial value for $\gamma_{f}$ and $\gamma_{r}$. In the following, we will refer to $\gamma$ as the system-bath coupling constant, irrespective of the simulation method, whether it is QTB, adQTB-r or adQTB-f. However, it should be stressed that $\gamma$ plays a different role in each of the three cases.

In the remaining of this section, we describe and compare the main features of the two variants of the proposed adaptive approach. A detailed description of the algorithm to integrate the generalized Langevin equation and of the self-tuning procedure for the system-bath coupling coefficients is provided in appendices $\mathrm{A}$ and $\mathrm{B}$, and summarized in Fig.11.

In the adQTB-r method, the friction force is in fact Markovian, with an expression similar to that of the standard QTB. In order to adapt $\gamma_{r}$, the QTB trajectory is decomposed into a sequence of segments. At the end of each segment, the spectra $C_{v v}(\omega)$ and $C_{v F}(\omega)$ are estimated from the previous segment of trajectory. The function $\gamma_{r}(\omega)$ is then adapted for each frequency in order to reduce $\Delta_{F D T}$ in the following of the simulation.

In the adQTB-f method, $\gamma_{f}$ depends on $\omega$ and as a consequence the memory kernel $\tilde{\gamma}_{f}(\tau)$ is not a $\delta$-function: the friction is non-Markovian. To compute it and integrate the generalized Langevin equation (15) efficiently, we use an extended phase-space approach in which $\gamma_{f}(\omega)$ is decomposed as a sum of Lorentzian terms. This decomposition also provides a way to evaluate $\Delta_{F D T}$ and adapt the value of $\gamma_{f}(\omega)$ at each time step of the simulation (see appendix B 2).

Note that, while $\gamma_{r}(\omega)$ is real by definition, $\gamma_{f}(\omega)$, defined as the Fourier transform of the friction kernel $\tilde{\gamma}_{f}(\tau)$, is in general complex. In fact, since $\tilde{\gamma}_{f}(\tau)$ is causal, the real and imaginary parts of its Fourier transform are related by Kramers-Kroning relations and the only case in which $\gamma_{f}(\omega)$ is real is when it is independent of $\omega$, which corresponds to the standard Markovian friction case. In the adQTB-f method, it is the real part $\operatorname{Re}\left[\gamma_{f}(\omega)\right]$ that should be adapted, since it is responsible for energy dissipation. However, modifying the $\operatorname{Re}\left[\gamma_{f}(\omega)\right]$ implies the presence of a non-zero imaginary part $\operatorname{Im}\left[\gamma_{f}(\omega)\right]$, which causes frequency shifts in the adQTB spectra. If these shifts are large, the dynamical properties obtained via adQTB-f might be significantly altered. Therefore in practical applications, $\operatorname{Im}\left[\gamma_{f}(\omega)\right]$ should be computed (using the formula in appendix A) and compared to the width of the relevant spectral features to evaluate its influence.

\section{B. Two-oscillator system}

We first illustrate the performance of the adQTB method on the two-oscillator model system described by eq. (14). Fig. 2.a and 2.b report the average energy in each harmonic oscillator obtained in QTB simulations as a function of $c_{3}$ and $c_{4}$, respectively (see details in the figure captions). The numerical solution of the Schroedinger equation provides an exact reference for this system (black lines in Fig. 2) and shows that the average energies should be almost independent of $c_{3}$ and $c_{4}$ in the range of values explored here. However, due to the ZPEL, the QTB results (shown in blue) are strongly sensitive to the anharmonicity: when the mode coupling is increased (via $c_{3}$ or $c_{4}$ ), the energy in the high-frequency mode decreases and the energy in the low-frequency mode increases.

In contrast, in both adQTB variants (green and red curves), nearly perfect compensation for the ZPEL is obtained over the whole range of coupling parameters using $\gamma=0.02$. This value is much smaller than the one needed to reach the correct quantum energy distribution in standard QTB (see section III C). The value $\gamma=0.01$ also provides satisfactory results except for the adQTB-r method in the case of strong anharmonicity, as analyzed below.

Fig. 3 shows the converged value of the adapted $\operatorname{Re}\left[\gamma_{f}(\omega)\right]$ and $\gamma_{r}(\omega)$, used to restore the quantum FDT in the adQTB method. As expected, in adQTB-r, $\gamma_{r}(\omega)$ is reduced at low frequencies and increased at high frequencies, while the reverse happens for $\operatorname{Re}\left[\gamma_{f}(\omega)\right]$ in adQTB-f. In both cases, the adaptation is driven by the discrepancy from the FDT, therefore $\gamma_{f}(\omega)$ and $\gamma_{r}(\omega)$ are 

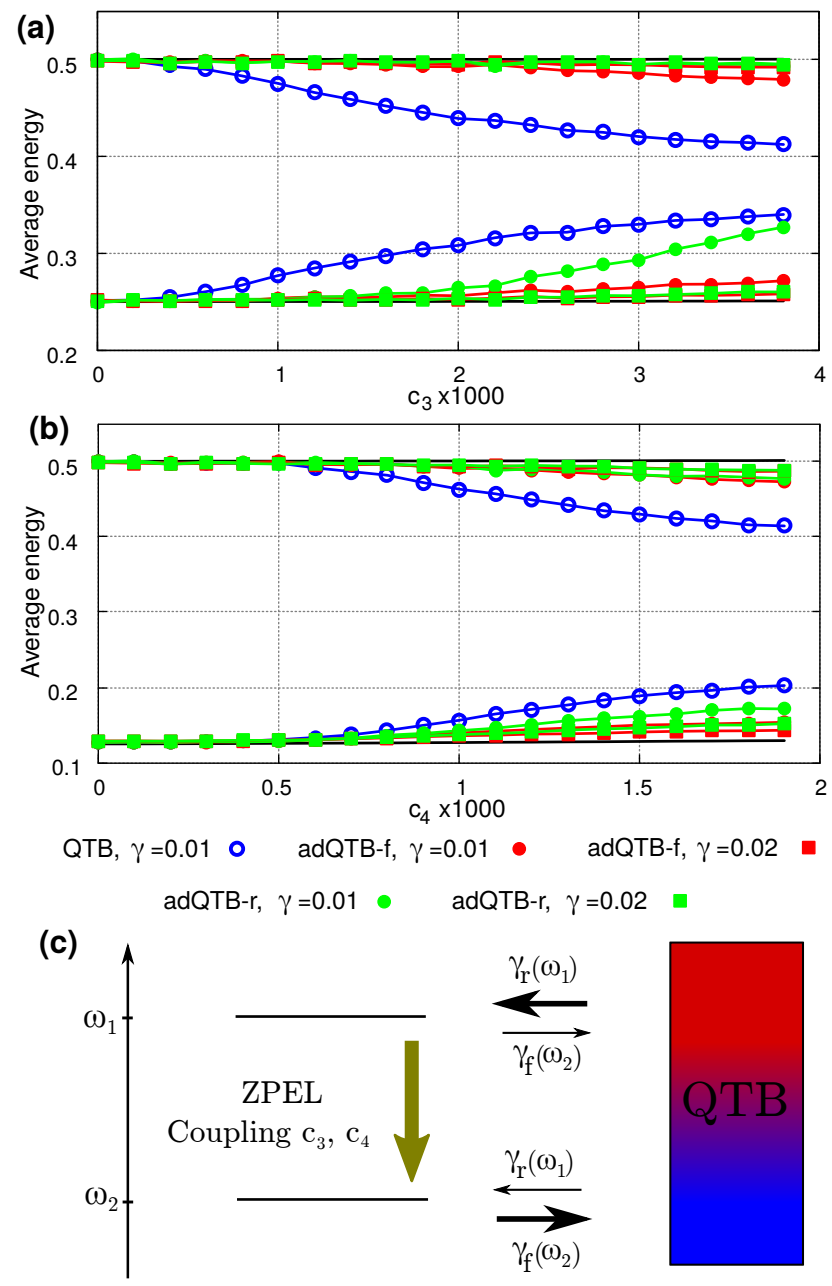

FIG. 2. Panels a and b: Average total energy in each harmonic oscillator of the model of equation (14), as a function of the coupling coefficients $c_{3}$ and $c_{4}$, for QTB and different versions of adQTB. The value of $\omega_{1}$ is fixed to 1 . In panel (a), $\omega_{2}=0.5$ and $c_{4}=0$ while $c_{3}$ varies. In panel (b), $\omega_{2}=0.25$, $c_{3}=0$ and $c_{4}$ is varied. Other simulation parameters are chosen as in Fig. 1. The different curves correspond to different values of the system-bath coupling constant $\gamma$ and the black lines show the exact result obtained through a numerical solution of the Schroedinger equation.

The panel $\mathrm{c}$ is a schematic representation of the adQTB scheme with adapted $\gamma_{r}$ and $\gamma_{f}$.

only modified close to the resonance frequencies $\omega_{1}$ and $\omega_{2}$ and hardly affected otherwise.

Despite the on-the-fly adaptation of $\gamma_{f}(\omega)$ and $\gamma_{r}(\omega)$, the choice of the coupling constant $\gamma$ is not indifferent. In particular, in the adQTB-r method the condition $\gamma_{r}(\omega)>0$ must hold, but it may not be always achievable in practice if $\gamma$ is chosen too small. Indeed, the algorithm tends to reduce $\gamma_{r}\left(\omega \simeq \omega_{2}\right)$ - close to the low-frequency mode - until the FDT is fulfilled. If $\gamma_{r}\left(\omega \simeq \omega_{2}\right)$ vanishes before this condition is met, eq. (18) becomes inoperative, and the ZPEL is not fully compensated for. This is why the low-frequency mode energy in Fig 2.a and 2.b
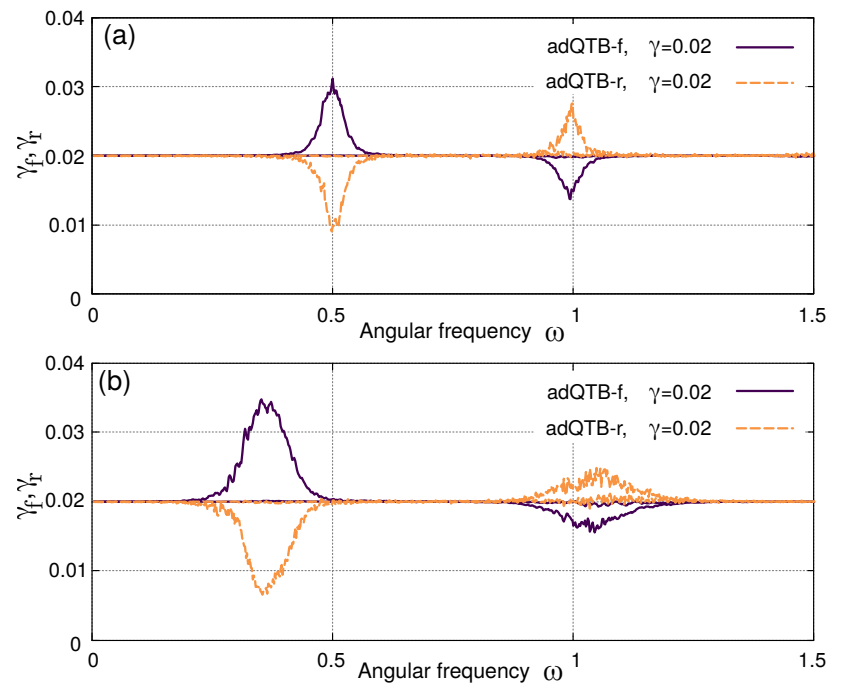

FIG. 3. Adapted value of the coefficients $\operatorname{Re}\left[\gamma_{f}(\omega)\right]$ and $\gamma_{r}(\omega)$ for the two-oscillator model with $c_{3}=3.8 \times 10^{-3}$ (panel a) and $c_{3}=1.9 \times 10^{-3}$ (panel $\mathrm{b}$ ). On each panel, curves of the same color are used for the coefficients for the oscillators 1 and 2, which are easily distinguished as the changes in the system-bath coefficients mostly occur close to the resonance frequencies $\omega_{1}$ and $\omega_{2}$ respectively. All the curves presented correspond to $\gamma=0.02$.

is markedly overestimated by the adQTB-r method for $\gamma=0.01$, whereas it becomes correct for $\gamma=0.02$. Although the adQTB-f variant seems less affected by this limitation, since $\operatorname{Re}\left[\gamma_{f}(\omega)\right]$ can in principle become negative, this algorithm too will eventually fail for very small values of $\gamma$. In practical applications of the method, it is thus important to check that the adaptation of the coefficients actually reduces $\Delta_{F D T}(\omega)$ to values close to zero for all angular frequencies $\omega$. A non-zero $\Delta_{F D T}(\omega)$ or a vanishing $\gamma_{r}(\omega)$ (in the adQTB-r method) indicate that the constant $\gamma$ should be increased to enable full compensation for the ZPEL.

\section{Energy fluctuations}

Computing the energy fluctuations is an even more stringent test for the adQTB method, since they are related to the fourth-order moments of the position and momentum probability distributions, on which the FDT criterion does not provide any direct information. For the two-oscillator model, we show in Fig. 4.a and 4.b the root mean square deviation (RMSD) of the energy in the lowfrequency mode, as a function of $c_{3}$ and $c_{4}$. The results obtained in adQTB are systematically improved with respect to standard QTB, but the energy fluctuations still tend to be slightly overestimated when the anharmonicity is strong, even in some cases in which the average energy is efficiently corrected from the ZPEL (see Fig 2). The high-frequency mode is not affected by this effect and its energy RMSD computed in adQTB agrees with 
the reference.

This result shows that the adQTB remains an approximate method: although the strict enforcement of the FDT allows compensating the effect of the ZPEL on the average energies, it does not guarantee that all moments of the equilibrium probability distribution are correct. However, the discrepancy between the adQTB and the exact energy RMSD only appears when a substantial part of the energy in the low-frequency mode comes from the ZPEL: when $\gamma$ is increased from 0.02 to 0.04 , the interaction with the bath is strengthened and the relative importance of the ZPEL reduced, so that the adQTB result for the RMSD is improved. Therefore, an appropriate choice of $\gamma$ (potentially larger than required to merely correct the average energy) should in general allow for a satisfactory simulation of the energy fluctuations.
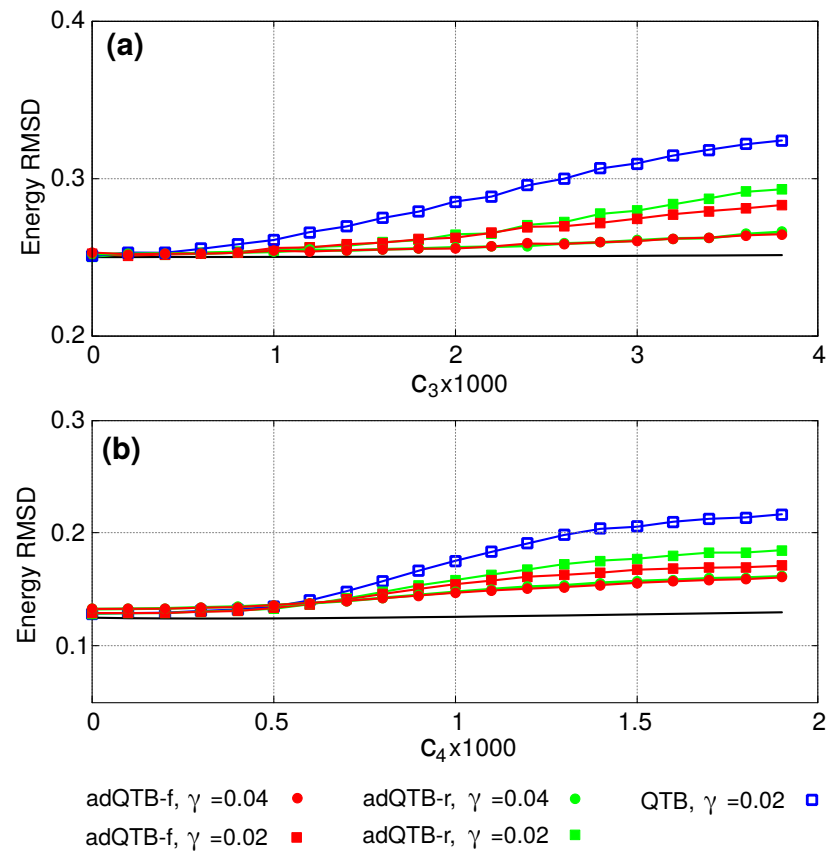

FIG. 4. RMSD of the total energy in oscillator 2 as a function of the anharmonicity parameters $c_{3}$ (panel a) and $c_{4}$ (panel b), as obtained in QTB and adQTB. All simulation parameters are similar to Fig 2 . The results are compared to the reference value (black line) obtained from the numerical solution of the Schroedinger equation.

\section{Numerical overhead compared to standard QTB}

The adQTB approach presents two types of numerical overhead with respect to the standard QTB. Firstly, the FDT criterion must be evaluated and the coefficients $\gamma_{f}(\omega)$ or $\gamma_{r}(\omega)$ must be tuned to ensure that it is satisfied for each frequency. The associated numerical cost is very limited in the case of the adQTB-r variant, about $5 \%$ overhead compared to standard QTB in our current implementation (for an equal number of time steps). In the case of adQTB-f, the implementation of the non-Markovian friction force requires introducing and propagating in time a set of auxiliary variables (see appendix A). The number of auxiliary variables is typically a few hundreds for each degree of freedom in the system. They obey a very simple evolution equation that does not involve the interatomic potential or its derivatives. The numerical overhead associated to the time propagation of the auxiliary variables is therefore substantial when interatomic interactions are represented via inexpensive analytical potentials (for example, adQTB$\mathrm{f}$ is about 200 times slower than standard QTB for the two-oscillator model), but this extra cost becomes essentially negligible in the case of first-principle molecular dynamics. The second source of numerical overhead is the fact that adQTB trajectories must include an adaptation time during which the coefficients $\gamma_{r}$ or $\gamma_{f}$ are adjusted (see appendices). We expect the associated computational cost to depend on the system under study: in the two-oscillator model presented above, we noticed that the random force spectrum $C_{v F}(\omega)$ becomes increasingly noisy when the anharmonicity is increased. Therefore a longer adaptation time is required to converge the system-bath coupling coefficients when $c_{3}$ or $c_{4}$ is large. In the most anharmonic cases, we estimated that the minimum number of adaptation time steps needed to converge the coefficients $\gamma_{f}$ or $\gamma_{r}$ is around $1-3 \times 10^{5}$. This figure is of the same order as the number of time steps required in standard QTB to converge the average energies with enough precision to obtain an accurate estimation of the ZPEL. However, in this work, we wish to provide a proof of principle for the adQTB scheme and we did not attempt a systematic study and optimization of the adaptation procedure, which is detailed in appendix B. In this appendix we provide some hints that might allow to speed-up the adaptation substantially and that will be pursued in further work. In the realistic applications presented in section $\mathrm{V}$, long trajectories are required (whether in classical MD, QTB or adQTB simulations) in order to reach convergence on the relevant statistical indicators. Therefore we were able to use a slow adjustment of the system-bath coupling coefficients and a long adaptation time, while retaining a relatively limited numerical overhead compared to standard QTB simulations.

\section{APPLICATION TO REALISTIC SYSTEMS}

In this section we experiment the adQTB method on two realistic systems that are known to exhibit significant ZPEL during QTB simulations, i.e. a model aluminium crystal ${ }^{31,32}$, and small clusters of neon ${ }^{33}$. In the $\mathrm{Al}$ crystal, the ZPEL results in an unbalance in the effective kinetic energy per mode with respect to the expected quantum result. An even more striking effect can be observed in the simulation of the solid-liquid phase transition of small clusters of rare gas, neon in particu- 
lar. In that case, the ZPEL destabilizes the geometrical structure of the cluster and dissociates the weak Van der Waals bonds, leading to a large error in the evaluation of the phase transition temperature or even suppressing the transition completely.

The modus operandi for these more realistic simulations is similar to the one employed in the previous section: a random force kernel $\gamma_{r, k}(\omega)$ and a dissipative kernel $\gamma_{f, k}(\omega)$ are associated to each degree of freedom $k$. In the adQTB-r (resp. adQTB-f) method, the coefficients $\gamma_{r, k}(\omega)$ (resp. $\left.\gamma_{f, k}(\omega)\right)$ are slowly adjusted all along the adQTB dynamics in order to compensate for the ZPEL, while the $\gamma_{f, k}(\omega)$ (resp. $\gamma_{r, k}(\omega)$ ) are kept constant and equal to $\gamma$. The adjustment is performed independently for each degree of freedom $k$, according to the corresponding FDT. The relevant observables are computed after an adaptation time sufficient to reach convergence on the system-bath coupling coefficients (see appendix B for more details on the adaptation procedure).

\section{A. Aluminium crystal}

QTB and adQTB molecular dynamics were carried out on a fcc crystal made of $N=256$ atoms at $T=10 \mathrm{~K}$. The atom-atom interaction is described by a Lennard-Jones pair potential:

$$
V\left(r_{i j}\right)=\sum_{i=1}^{N} \sum_{j>i}^{N} 4 \epsilon\left(\left(\frac{\sigma}{r_{i j}}\right)^{12}-\left(\frac{\sigma}{r_{i j}}\right)^{6}\right)
$$

with $\epsilon / k_{B}=1450.6 \mathrm{~K}, \sigma=2.54 \AA$, as in Ref. 31 and 32. Interactions between particles with interatomic distance $r_{i j} \geq r_{c u t}=2.49 \sigma \quad$ were neglected. We used periodic boundary conditions with a box length of $4 \sqrt{2} \sigma$. In the QTB dynamics, we used a time step of $5 \mathrm{fs}$, a coupling constant $\gamma=2 \mathrm{THz}$, and a frequency cutoff $\omega_{\text {cut }}=128 \times 10^{12} \mathrm{rad} / \mathrm{s}$. The adQTB trajectories consist of 3000 segments of 4000 steps each. Only the last 1500 segments are used to compute statistical averages, in order to allow for the prior adaptation of the system-bath coupling coefficients $\gamma_{r}(\omega)$ or $\gamma_{f}(\omega)$.

Previous QTB studies of this system ${ }^{31,32}$ relied on normal mode analysis and the harmonic approximation in order to evaluate the ZPEL. Fig. 5 shows the effective temperature of the normal modes (i.e. acoustic phonon modes) as a function of their frequency, as obtained with the standard QTB and adQTB methods. This effective temperature is proportional to the average kinetic energy per mode, computed by re-expressing the atomic velocities in the normal mode basis. Following Refs 31 and 32 , the effective temperature is compared to $\theta(\omega, T) / k_{B}$, which is the result expected from the quantum harmonic approximation. At this low temperature, $T=10 \mathrm{~K}$, the kinetic energy in the normal modes consists almost exclusively of zero-point energy.

The standard QTB result exhibits significant ZPEL: the kinetic energy in the high-frequency modes (above

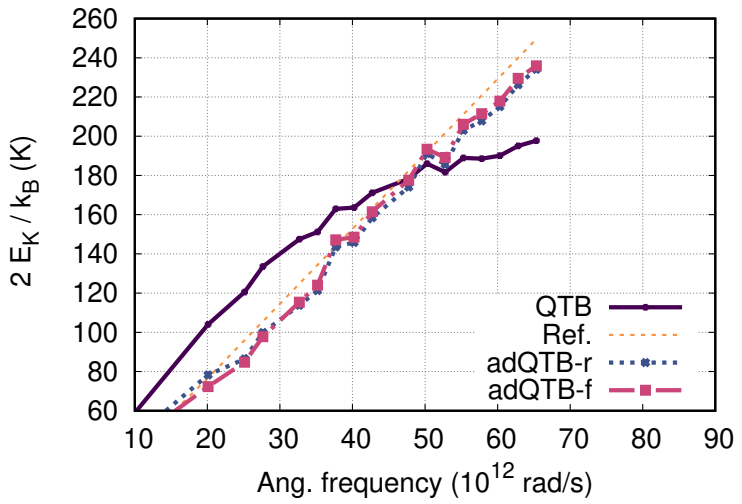

FIG. 5. Al crystal: Effective normal mode temperature computed from the average kinetic energy per mode, $2 E_{K} / k_{B}$. The results obtained from QTB, adQTB-r, and adQTB-f simulations are compared to the reference $\theta(\omega, T) / k_{B}$, expected from the quantum harmonic approximation.

$50 \times 10^{12} \mathrm{rad} / \mathrm{s}$ ) is lower than expected from the harmonic approximation while the kinetic energy in the low frequency modes is higher than the reference. The use of either adQTB-r or adQTB-f restores the reference quantum distribution almost exactly. Both adQTB-r and adQTB-f reach similar results, confirming the accuracy and robustness of the adaptive procedure.

The results of the normal mode analysis are consistent with the those obtained from the average deviation from the fluctuation-dissipation theorem, $\Delta_{F D T}(\omega)$, which are presented in Fig. 6.a. In the standard QTB simulation, $\Delta_{F D T}(\omega)$ assumes large positive and negative values, changing sign around $50 \times 10^{12} \mathrm{rad} / \mathrm{s}$. In contrast, in both adQTB variants, this difference is close to zero on the whole frequency range, confirming that the adaptation of the system-bath coupling coefficients is successful in enforcing the FDT and therefore in compensating for the ZPEL. The value of $50 \times 10^{12} \mathrm{rad} / \mathrm{s}$ for the sign change is consistent with the results of Fig. 5.

It is worth stressing that the FDT criterion is more general than the normal mode analysis since it does not rely on the harmonic approximation. This approximation is relevant here because at this low temperature, the system only explores configurations of relatively weak anharmonicity but it cannot be used as a completely general tool. The vibrational density of states (DOS) displayed in Fig. 6.b shows that both adQTB-r and adQTB-f redistribute the energy from low frequencies to high frequencies with respect to the standard QTB results.

Fig. 7 shows the converged $\gamma_{r}(\omega)$ (adQTB-r, panel a) and $\gamma_{f}(\omega)$ (adQTB-f, panel b) coefficients that allow for ZPEL compensation. These quantities display the expected behavior. Panel (a) shows that, starting from the initial value $\gamma=2 \mathrm{THz}$, the coefficient $\gamma_{r}(\omega)$ adapted with adQTB-r decreases at low frequencies, with a minimum value around $\omega \simeq 20 \times 10^{12} \mathrm{rad} / \mathrm{s}$, and it increases at high frequencies with a maximum around 
(a)

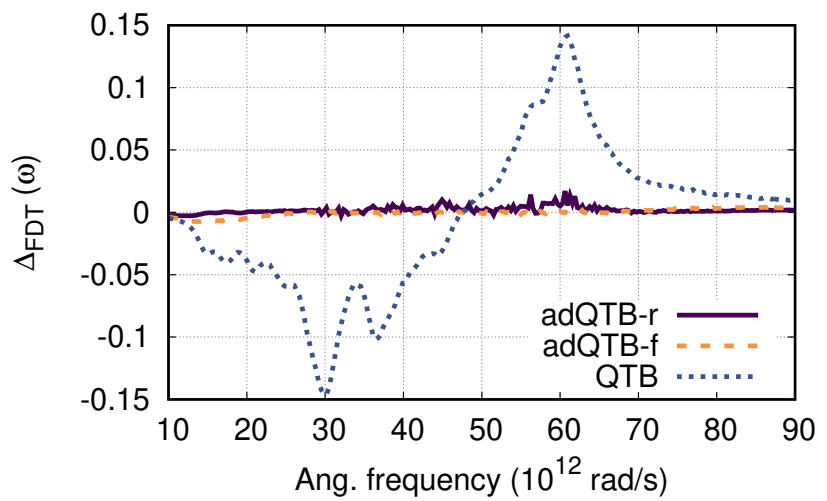

(b)

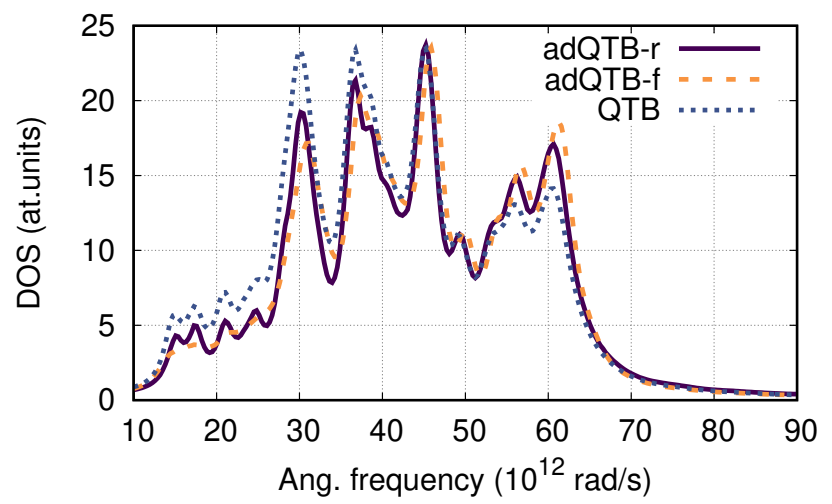

FIG. 6. Panel (a) : Deviation $\Delta_{F D T}(\omega)$ from the fluctuationdissipation theorem for QTB, adQTB-r and adQTB-f of the $\mathrm{Al}$ crystal. The deviation is normalized to the maximum value of $m \gamma_{r}(\omega) C_{v v}(\omega)$. Panel (b) : vibrational density of states obtained from the velocity autocorrelation spectra $m C_{v v}(\omega) / \theta(\omega, T)$.

$\omega \simeq 70 \times 10^{12} \mathrm{rad} / \mathrm{s}$. The angular frequency for which $\gamma_{r}(\omega)$ is maximum (resp. minimum) is close to that of the highest (resp. lowest) normal modes of the system. On the other hand, Fig. 7.b shows that the friction coefficient $\operatorname{Re}\left[\gamma_{f}(\omega)\right]$, adapted with adQTB-f, follows the opposite behavior: it increases below $50 \times 10^{12} \mathrm{rad} / \mathrm{s}$ and decreases above this angular frequency, with variations similar in magnitude to that of $\gamma_{r}(\omega)$. The imaginary part of the dissipative kernel $\operatorname{Im}\left[\gamma_{f}(\omega)\right]$ also varies, taking positive values at very low frequencies and negative values as low as $-1 \mathrm{THz}$ on most of the relevant frequency range. This imaginary part induces small shifts of the peaks in the vibrational DOS (of the order of $0.5 \times 10^{12} \mathrm{rad} / \mathrm{s}$ ), apparent in the slight difference between the adQTB-r and adQTB-f spectra in Fig. 6.b (see also appendix A). In spite of these small discrepancies, the spectra obtained with the two adQTB variants display an overall good agreement, indicating that the spurious dynamical effects introduced by the adQTB method (particularly adQTBf) are limited. This is because the adapted friction coef- (a)

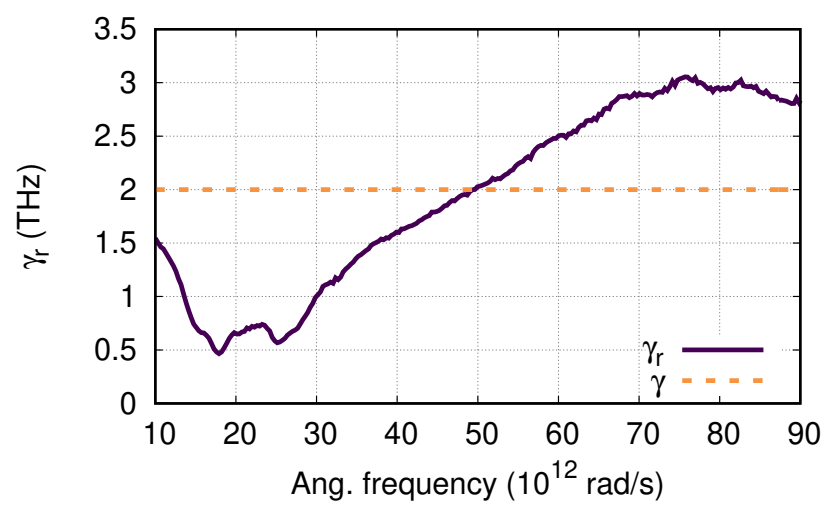

(b)

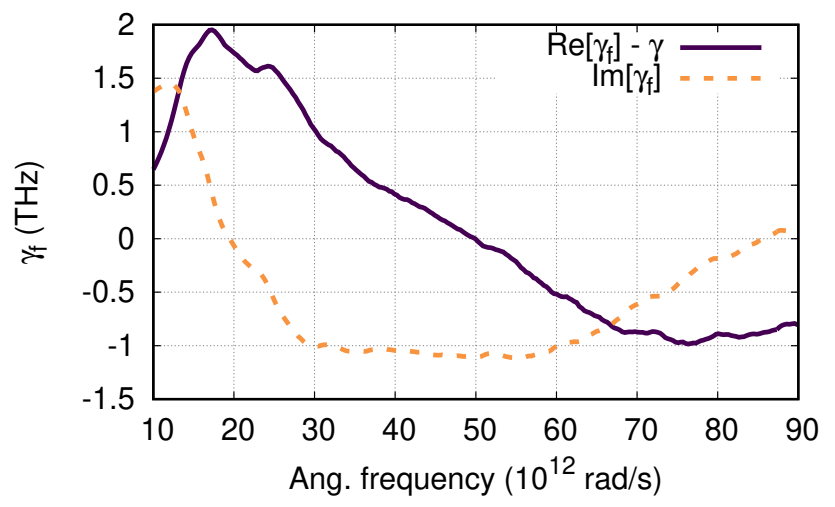

FIG. 7. Panel (a) : converged value of $\gamma_{r}(\omega)$ adapted with adQTB-r, starting from the initial value $\gamma=2 \mathrm{THz}$. Panel (b) : converged value of $\operatorname{Re}\left[\gamma_{f}(\omega)\right]$ and $\operatorname{Im}\left[\gamma_{f}(\omega)\right]$ adapted with adQTB-f, starting from the same initial value. The figure shows only the non-Markovian part, $\gamma_{f}(\omega)-\gamma$, of the friction kernel (see appendix A). In both panels, the coefficients $\gamma_{r, k}(\omega)$ and $\gamma_{f, k}(\omega)$ are averaged over all $3 N$ equivalent degrees of freedom $k$ of the system.

ficient remains small compared to the angular frequency of the relevant vibrational modes of the system. The coupling constant $\gamma=2 \mathrm{THz}$ is also considerably smaller than those required to limit the ZPEL in standard QTB calculations: it should be compared, for instance, with the coupling used in Ref. 32 to reinstate the correct average energy per mode, i.e. $\gamma=10 \mathrm{THz}$.

\section{B. The $\mathrm{Ne}_{13}$ cluster}

A recent study ${ }^{33}$ pointed out the consequences of the zero-point energy leakage in QTB simulations of lowtemperature phase transitions in Lennard-Jones clusters. The results obtained for the solid-liquid transition in these systems were shown to be highly sensitive to the ZPEL. Indeed, the QTB provides a satisfactory (though slightly underestimated) estimate for the transition tem- 
perature in the case of argon clusters, but it is completely unable to describe the transition in the case of neon clusters: due to ZPEL, the QTB simulations predict an abnormal liquid-like phase even at the lowest temperatures. Due to this strong influence of the ZPEL, and considering the low temperatures involved and the large anharmonicity of the weak inter-atomic forces, neon clusters are a challenging test case for the adaptive QTB method.

In order to characterize the solid-liquid transition in Ne clusters, we focus primarily on the pair correlation function $g(r)$, whose profile changes significantly between the two phases.

We also present the results obtained for the Lindemann index $\delta_{L}$, defined from the root-mean-square bond length fluctuation averaged on all atomic bonds in the cluster:

$$
\delta_{L}=\frac{2}{N(N-1)} \sum_{i=1}^{N} \sum_{j>i}^{N} \frac{\sqrt{\left\langle r_{i j}^{2}\right\rangle-\left\langle r_{i j}\right\rangle^{2}}}{\left\langle r_{i j}\right\rangle}
$$

The Lindemann index is commonly used to pinpoint solid-liquid phase transitions ${ }^{33,41}$, however, as we will show, its interpretation in the present context is not straightforward and should be complemented by the study of the pair correlation function.

\section{Parameters}

We carried out simulations for the $\mathrm{Ne}_{13}$ cluster, placing the atoms in a starting configuration of $I_{h}$ symmetry. A Lennard-Jones pair potential is used to describe interatomic interactions with the parameters $\epsilon / k_{B}=34.9 \mathrm{~K}$, $\sigma=2.78 \AA$, as in Ref. 33. A confining potential of the form:

$$
V_{\text {conf }}\left(r_{i j}\right)=\sum_{i=1}^{N} \sum_{j>i}^{N} \epsilon\left(r_{i j}-r_{r e f}\right)^{4}
$$

is added to avoid cluster vaporization, with $r_{r e f}=10 \AA$. We compare classical Langevin (LGV), standard QTB and both adQTB methods (adQTB-r and adQTB-f) for a range of temperatures between 1 and $18.3 \mathrm{~K}$. We use a coupling constant $\gamma=2 \mathrm{THz}$, a time step $\Delta t=1 \mathrm{fs}$ and the angular frequency cutoff ${ }^{38}$ is fixed to $\omega_{\text {cut }}=$ $63 \times 10^{12} \mathrm{rad} / \mathrm{s}$. In this system, the convergence of the relevant statistical quantities (particularly $\delta_{L}$ ) requires very long dynamics, therefore the adQTB adaptation time (see sec. IV D) has a minor impact on the efficiency of the simulation and a slow adjustment of the systembath coupling coefficients can be used. More precisely, the trajectories consist of a sequence of 30000 segments of 16000 steps each. The first 1250 segments are used to slowly adjust the set of coefficients $\gamma_{r}(\omega)$ and $\gamma_{f}(\omega)$. Reference results are obtained with path integral molecular dynamics (PIMD) simulations that consist of $4 \times 10^{8}$ steps of $1 \mathrm{fs}\left(5 \times 10^{5}\right.$ of which are used for thermalization). The PIMD simulations use 32 beads at all temperatures. The convergence with the number of beads was checked for the lowest temperature case $(2.5 \mathrm{~K})$ : using 64 beads (and a shorter timestep) at this temperature did not produce noticeable changes in the observables.

\section{Results}

A comparison between the results obtained by the different simulation techniques for the pair correlation function $g(r)$ is shown in Fig. 8 at a temperature $T=4 \mathrm{~K}$, just below the expected transition temperature. First, one can notice the striking difference between the classical Langevin and the reference PIMD simulations. The classical result (denoted LGV on Fig. 8) displays sharp peaks at $2.8,4.6$ and $5.4 \AA$, corresponding to the first, second and third nearest-neighbor distances respectively. In the quantum PIMD simulations, these peaks are significantly broadened (the third one remaining only as a shoulder to the second) and slightly shifted to $2.9,4.8$ and $5.6 \AA$. This broadening of the peaks is characteristic of the quantum delocalization of the neon nuclei and the difference between the classical and the quantum results shows that nuclear quantum effects must be taken into account in order to describe correctly the system at such low temperatures.

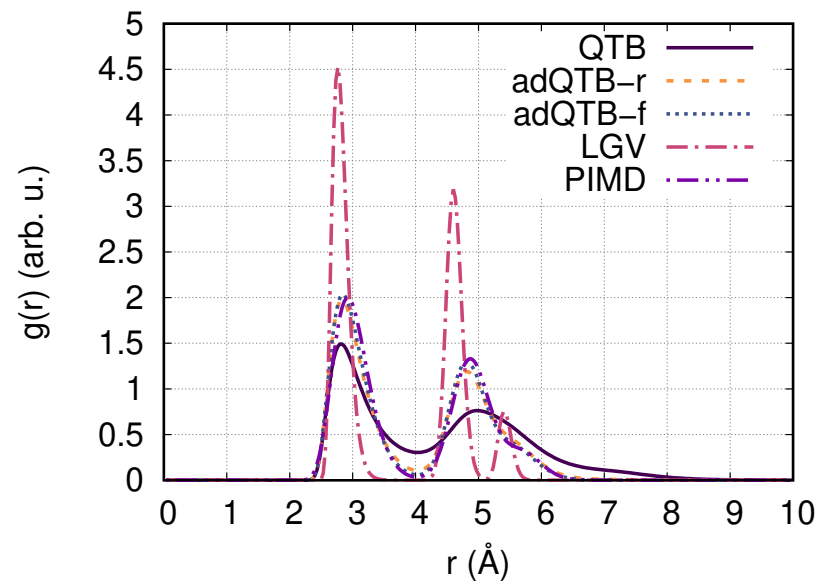

FIG. 8. $\mathrm{Ne}_{13}$ cluster pair correlation function $g(r)$ computed with Langevin (LGV), standard QTB, adaptive random QTB (adQTB-r), adaptive friction QTB (adQTB-f). The temperature is $T=4 \mathrm{~K}$ and the coupling constant is $\gamma=2 \mathrm{THz}$. Reference results are obtained from path integral molecular dynamics (PIMD).

All QTB and adQTB pair correlation functions present broader peaks than the classical Langevin results, indicating that the zero-point energy introduced by the colored noise increases the nuclear delocalization, as expected. However, the QTB result presents a profile characteristic of the abnormal liquid-like phase that was reported in Ref. 33: the peaks of $g(r)$ are much broader than those of the PIMD reference, and the second and 
third neighbor distances become indistinguishable. Crucially, around $r=4 \AA$, between the first and second neighbor distances, the minimum of $g(r)$ is non-zero, in sharp contrast with the PIMD reference. This is a signature of the loss of the ordered structure and a consequence of the important atom diffusion occurring in the liquid-like phase. This is due to the ZPEL, which causes an unphysical excess of energy in the low frequency modes, spuriously breaking the weak atomic bonds in the system. This abnormal liquid-like state persists in the standard QTB simulations down to $1 \mathrm{~K}$ (the lowest temperature explored in this work). Figure 8 shows that, remarkably, both adQTB variants restore the proper physical behavior: the pair correlation functions obtained are in excellent agreement with the PIMD simulations. The most significant difference between the adQTB and the reference curve is that, around $r=4 \AA$, the minimum value of $g(r)$ is slightly overestimated by both adQTB methods. This small discrepancy (hardly visible in Fig. 8) is most probably related to the effect noted in section IV C: even when successful in correcting the average energy per mode, the adQTB method may slightly overestimate the energy fluctuations in the low-frequency modes.

To complete this analysis, we display on Fig. 9.a the average deviation from the fluctuation-dissipation theorem $\Delta_{F D T}(\omega)$ obtained in QTB and in adQTB. The $\Delta_{F D T}(\omega)$ curve is close to zero for both adaptive variants, showing that the adaptive scheme successfully compensates for the massive ZPEL observed in standard QTB simulations. Fig. 9.b, shows the corresponding adapted system-bath coupling coefficients at temperature $T=4 \mathrm{~K}$ and, for comparison, at $T=18.3 \mathrm{~K}$ (i.e. above the expected solid-liquid transition). In adQTB-r simulations, for high frequencies (above $\omega=7 \mathrm{Trad} / \mathrm{s}$ ), $\gamma_{r}(\omega)$ increases from its starting value $\gamma=2 \mathrm{THz}$, while it decreases for low frequencies. Its minimum value is approximately $0.5 \mathrm{THz}$ at $T=4 \mathrm{~K}$. This result indicates that, in this case, $\gamma$ should be at least 2 THz: since $\gamma_{r}(\omega)$ only takes positive values, a smaller $\gamma$ would lead to a residual ZPEL that the adQTB procedure would not fully compensate for, as discussed in section IV. The adapted dissipative kernel $\operatorname{Re}\left[\gamma_{f}(\omega)\right]$ shows the opposite trend: it increases below $7 \times 10^{12} \mathrm{rad} / \mathrm{s}$ and decreases above this angular frequency. The main departure from the symmetric behavior of the system-bath coupling coefficients in the two adQTB variants is that, contrary to $\gamma_{r}(\omega)$ that reaches a minimum at $\omega=2 \times 10^{12} \mathrm{rad} / \mathrm{s}$, the friction coefficient $\operatorname{Re}\left[\gamma_{f}(\omega)\right]$ tends to a maximum towards zero frequency. This difference is most probably a consequence of the modifications induced by the adQTB-f on the dynamical properties of the system (additional broadening of the vibrational modes and frequency-shifts due to the imaginary part $\left.\operatorname{Im}\left[\gamma_{f}\right]\right)$. At the higher temperature $T=18.3 \mathrm{~K}$, the cluster is liquid-like and the ZPEL has less impact. Therefore the variations of the systembath coupling coefficients are of smaller amplitude over the whole range of frequency, and even standard QTB
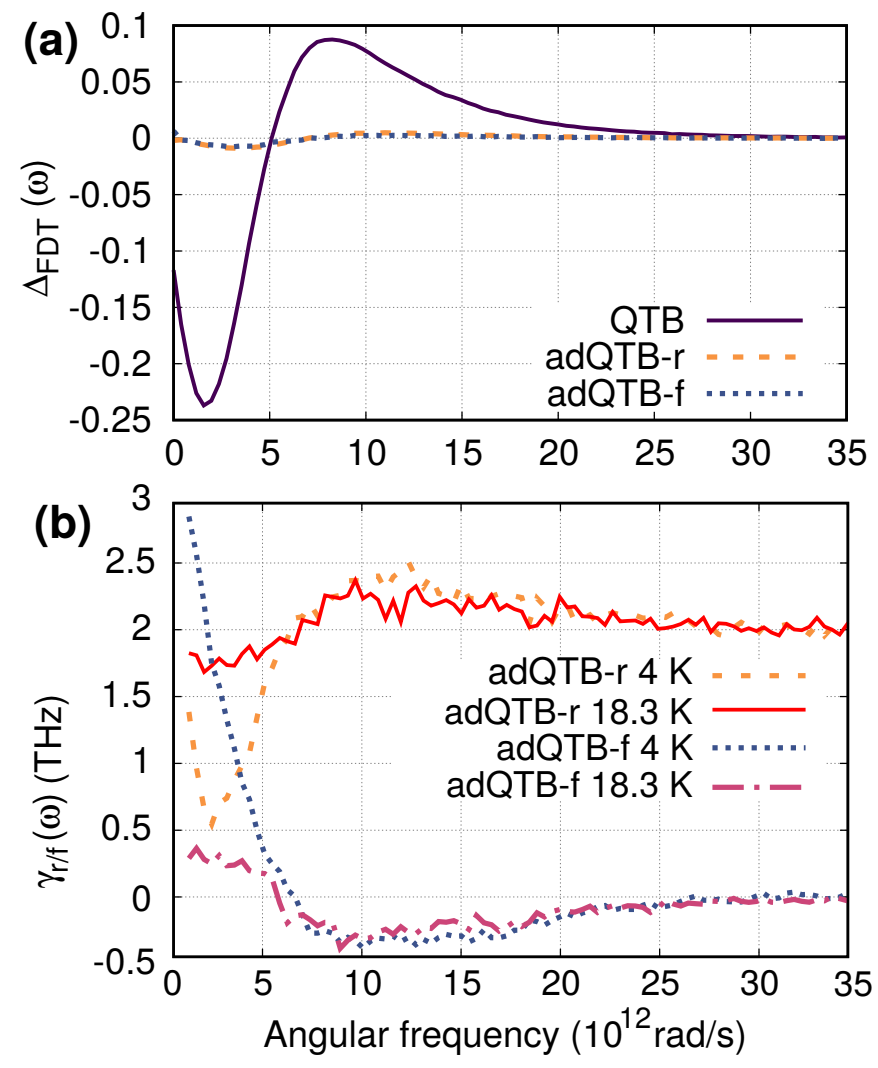

FIG. 9. Panel a: Deviation from the quantum fluctuationdissipation theorem $\Delta_{F D T}$ defined in eq. (18), as obtained with QTB and both adQTB variants at $T=4 \mathrm{~K}$ and $\gamma=2$ $\mathrm{THz}$ in the $\mathrm{Ne}_{13}$ cluster. Panel b : Set of adapted coefficients $\gamma_{r}(\omega)$ and $\operatorname{Re}\left[\gamma_{f}(\omega)\right]-\gamma$ obtained with adQTB-r and adQTBf, respectively. Two temperatures are represented: $T=4 \mathrm{~K}$ and $T=18.3 \mathrm{~K}, \gamma$ is fixed to $2 \mathrm{THz}$.

performs reasonably well at this temperature.

To conclude, we discuss the results obtained for the Lindemann index $\delta_{L}$, defined in eq. (20), which describes the thermal fluctuations of the atomic bond lengths. This parameter was used in particular in Ref. 33 to characterize the abnormal liquid-like phase induced by the ZPEL. The results are shown in fig. 10. We first compare the classical (LGV in Fig. 10) and the reference (PIMD) quantum results. For the classical nuclei, $\delta_{L}$ goes to zero linearly at low temperatures, whereas the PIMD result stabilizes around 0.07 due to the zero-point motion. In both cases a sharp increase of $\delta_{L}$ is observed between 5 and $7 \mathrm{~K}$, signalling the solid-liquid phase transition. As expected, this transition is shifted to a slightly lower temperature when nuclear quantum effects are included ${ }^{33}$. Figure 10 also shows that, due to the ZPEL, no such transition is observed in the standard QTB simulations. As it was already apparent from the results for $g(r)$, the cluster remains liquid-like down to the lowest temperatures simulated (the results for $g(r)$ at $T=1 \mathrm{~K}$ are qualitatively similar to that of Fig. 8 for $T=4 \mathrm{~K}$ ), consequently $\delta_{L}>0.3$ for all temperatures. In the case of the adQTB simulations, the interpretation of the Lindemann 


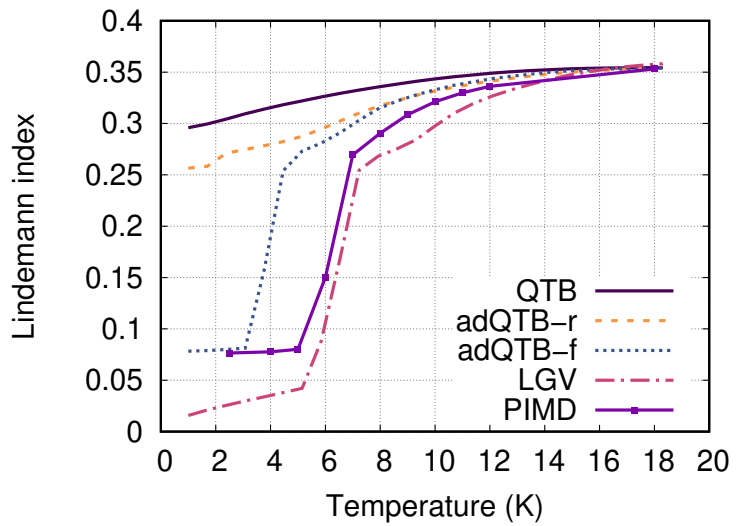

FIG. 10. Lindemann index $\delta_{L}$ from eq. (20), as a function of $\mathrm{T}$, computed from Langevin (LGV), standard QTB, adQTB$\mathrm{r}$ and adQTB-f for $\gamma=2 \mathrm{THz}$. Reference results are obtained from path integral molecular dynamics (PIMD).

curve is not straigthforward. We recall that, at $T=4 \mathrm{~K}$, the adQTB successfully corrects the ZPEL, and that the pair correlation function is close to that of the correct solid state, and very different from the standard QTB result. In spite of that, the Lindemann index obtained in adQTB at $4 \mathrm{~K}$ is significantly above the PIMD reference and closer to the values typical of the liquid-like phase. This apparent contradiction is explained as follows: although the adQTB simulations reproduce satisfactorily the overall structure of the cluster, they slightly overestimate the probability of the atomic diffusion events, by which two or more Ne atoms change sites. This overestimation also shows from the detailed analysis of the pair correlation function around $4 \AA$, where $g(r)$ is very small but non-zero, confirming that atoms can occasionally change site in the cluster. These events are rare but they strongly contribute to enhancing $\delta_{L}$ towards values close to that of the liquid-like phase, even though the average geometrical structure of the solid cluster is well described. Therefore $\delta_{L}$ cannot be used as a unique indicator of the phase transition which is more robustly described through the pair correlation function $g(r)$.

The value $\gamma=2 \mathrm{THz}$ that is used in this section is sizable compared with the typical vibration frequencies of the system. Such a large $\gamma$ is needed to fully compensate for the ZPEL due to the weakness and strong anharmonicity of the atomic bonds in the $\mathrm{Ne}_{13}$ clusters. However, the spectral broadening induced by $\gamma$ also affects the accuracy of the QTB results. In appendix C, we show that increasing $\gamma$ to $4 \mathrm{THz}$ in the adQTB simulations reduces the probability of the atomic diffusion events (consistently with the analysis of section IV C), and therefore improves the adQTB predictions for $\delta_{L}$. But increasing $\gamma$ also enhances the spectral broadening which in turn impacts the accuracy of the simulations. The optimal choice for $\gamma$ therefore implies a compromise, as discussed in more details in appendix $\mathrm{C}$.

\section{SUMMARY AND CONCLUSION}

Previous works on the problem of the ZPEL in simulations using a quantum thermal bath ${ }^{31-33}$ (QTB) have underlined the caution required when employing this method to approximate nuclear quantum effects in complex chemical and physical systems, and introduced the possibility of increasing the system-bath coupling to reduce the importance of the leakage. However, this approach to mitigate the ZPEL can have a strong impact on the statistical estimates. In this work, we aimed at understanding the ZPEL in more detail and reduce it substantially without increasing the system-bath coupling to large values.

First, we have established a quantitative connection between ZPEL and deviations from the quantum fluctuation-dissipation theorem (FDT). In the context of the QTB, the FDT can be expressed as a relation between the real part of the velocity random force correlation function and the velocity autocorrelation function: equation (13). This result is completely general and gives access to a quantitative estimate of the ZPEL without resorting to normal mode calculations or to comparisons with path integral simulations.

Second, we have proposed a new method in which the quantum FDT is actively enforced by adjusting the coupling between the quantum bath and the classical degrees of freedom. Rather than a constant $\gamma$, we have used a frequency-dependent coupling which is finely tuned all along the simulation, in order to suppress the ZPEL. This new type of bath, which we named adaptive QTB, can be realized in practice by tuning either the random force memory kernel (adQTB-r) or the dissipative memory kernel (adQTB-f). After detailed assessment on a system of coupled oscillators, the adQTB was experimented on two more realistic cases, testing the scalability and the accuracy of the new algorithms: a model aluminium crystal at low temperature and the more challenging problem of the solid-liquid phase transition in small neon clusters.

In the $\mathrm{Al}$ crystal, the FDT criterion provided an onthe-fly evaluation of the importance of the ZPEL, and the adQTB method allowed to compensate for the leakage as evidenced in the average energy distribution within the acoustic phonon modes. This study confirmed the applicability of the adQTB approach to anharmonic solids and extended systems. The $\mathrm{Ne}_{13}$ cluster is a particularly challenging case for the adQTB method: the potential is strongly anharmonic and the atomic bonds are weak, so that the system exhibits many imaginary frequencies in its mode analysis close to the melting temperature and above it. Nonetheless, both adQTB-f and adQTB-r methods were successful in compensating for the ZPEL at low temperature $(T=1 \mathrm{~K}$ to $18.3 \mathrm{~K})$. In particular, the new algorithms enabled recovering a solid-like cluster at low temperatures at which the standard QTB predicted an abnormal liquid-like phase.

In general, adQTB-f proved slightly more efficient than adQTB-r in compensating for the ZPEL. However, 
adQTB-f should be used with particular care, due to the possible frequency shifts induced by the imaginary part of the memory kernel. We noted that enforcing the FDT enables to compensate for the ZPEL and correct the first moment of the energy distribution. As discussed in the text, this does not guarantee that higher moments are accurately reproduced. Nonetheless, the general and systematic method proposed here substantially extends the range of possible applications of QTB-based approaches. The first tests performed in this work indicate that the numerical overhead of the adaptive algorithms compared to standard QTB can be kept relatively low. A notable feature of these algorithms is that the number of evaluations of the physical force per step is the same as in standard QTB, a crucial and promising property in view of applications involving first-principle potentials. Future work will consider improvements in the efficiency of our algorithms and a more detailed comparison with alternative (e.g. path integral) methods. A problem of particular interest will be the applicability of the adQTB to anharmonic liquids (flexible water models for instance) that can display strong ZPEL from the high-frequency intra-molecular vibration modes to the inter-molecular modes.

We conclude by observing that some of the concepts developed in this work might find application in other semi-classical methods that are also affected by the ZPEL, such as the quantum thermostat ${ }^{15,16}$ or techniques based on quantum averages over classical distributions ${ }^{12-14,42}$.

\section{ACKNOWLEDGEMENT}

Etienne Mangaud acknowledges the financial support from Labex MATISSE. We also thank Florent Calvo for sharing with us the parameters used in Ref. 33 for the neon clusters, and Sofiane Schaack for helpful discussions about the manuscript. The authors also acknowledge the Germaine De Staël exchange program for providing mobility funds that greatly facilitated the completion of this work. Support from the European Unions Horizon 2020 research and innovation program under the grant agreement No. 676531, project E-CAM, is also acknowledged.

\section{A. INTEGRATION OF THE GENERALIZED LANGEVIN EQUATION}

In this appendix we detail the numerical procedure adopted for the integration of the generalized Langevin equation (eq. (15) of the main text):

$$
m \dot{v}=-\frac{\partial V}{\partial x}-m \int_{0}^{\infty} \tilde{\gamma}_{f}(\tau) v(t-\tau) d \tau+F(t)
$$

Section A 1 describes the integration scheme used for the colored random force, which is common to the QTB and both adQTB variants, while the section A 2 presents the extended variable strategy that is used for the propagation of the generalized friction force in the implementation of the adQTB-f method. The integration algorithms are schematized in Figure 11, which also presents the additional steps introduced for the adaptation of the system-bath coupling coefficients (the adaptation procedure is described in detail in appendix B).

\section{Modification of the BAOAB algorithm for colored noise}

Our integration scheme is based on the BAOAB algorithm for the integration of the Langevin equation, which was shown to be remarkably accurate ${ }^{43,44}$. This section presents its generalization to the case of a colored random force as in the QTB dynamics.

The BAOAB algorithm is a time-splitting method: during a time step $\Delta t$, five successive steps of three different types (B, A and O) are applied to the phase space vector. Step A corresponds to the evolution of the positions owing to the momentum during a half-step $\frac{\Delta t}{2}$. Step $\mathrm{B}$ corresponds to the evolution of the momentum in the field of the conservative forces during $\frac{\Delta t}{2}$. Finally Step O is the one in which the velocity is propagated under the action of the friction and random forces, according to:

$$
\dot{v}(t)=-\gamma v(t)+\frac{F(t)}{m}
$$

In the case of a classical white-noise Langevin dynamics, the equation above corresponds to an OrnsteinUhlenbeck process and it can be integrated analytically. In the colored-noise case, it is still possible to integrate this block exactly by applying a timestep-dependent correction to the random force spectrum. To prove this, we first integrate equation (23) and write:

$$
v(t+\Delta t)=\mathrm{e}^{-\gamma \Delta t} v(t)+\underbrace{\frac{1}{m} \mathrm{e}^{-\gamma \Delta t} \int_{0}^{\Delta t} d \tau \mathrm{e}^{\gamma \tau} F(t+\tau)}_{R_{t}}
$$

We now consider the autocorrelation of the discretized 
noise $R_{t}$ :

$$
\begin{aligned}
& \left\langle R_{t} R_{t^{\prime}}\right\rangle \\
& =\frac{1}{m^{2}} \mathrm{e}^{-2 \gamma \Delta t} \int_{0}^{\Delta t} d \tau \int_{0}^{\Delta t} d \tau^{\prime} \mathrm{e}^{\gamma\left(\tau+\tau^{\prime}\right)}\left\langle F(t+\tau) F\left(t^{\prime}+\tau^{\prime}\right)\right\rangle \\
& =\frac{\mathrm{e}^{-2 \gamma \Delta t}}{2 \pi m^{2}} \int_{0}^{\Delta t} d \tau \int_{0}^{\Delta t} d \tau^{\prime} \mathrm{e}^{\gamma\left(\tau+\tau^{\prime}\right)} \int_{-\infty}^{+\infty} d \omega \mathrm{e}^{i \omega\left(t^{\prime}+\tau^{\prime}-t-\tau\right)} C_{F F}(\omega) \\
& =\frac{1}{2 \pi} \int_{-\infty}^{+\infty} d \omega \mathrm{e}^{i \omega\left(t^{\prime}-t\right)} \frac{1-2 \mathrm{e}^{-\gamma \Delta t} \cos (\omega \Delta t)+\mathrm{e}^{-2 \gamma \Delta t}}{\gamma^{2}+\omega^{2}} \frac{C_{F F}(\omega)}{m^{2}}
\end{aligned}
$$

where we used the time translation invariance of the random force autocorrelation function to derive the third line of the equation above. The last line shows that the discretized noise $R_{t}$ used in the propagation of step $\mathrm{O}$ should be generated with a correlation spectrum equal to the target spectrum $C_{F F}(\omega) / m^{2}$, given by eq. (2) for the QTB, multiplied by the correction factor:

$$
g(\omega)=\frac{1-2 \mathrm{e}^{-\gamma \Delta t} \cos (\omega \Delta t)+\mathrm{e}^{-2 \gamma \Delta t}}{\gamma^{2}+\omega^{2}}
$$

Note that in the limit $\Delta t \rightarrow 0$, the correction reduces to:

$$
g(\omega)=\Delta t^{2}+O\left(\Delta t^{3}\right)
$$

Thus in the small time step limit, $R_{t}$ has a spectrum similar to $F(t)$, except for a factor $\Delta t^{2}$ coming from the integration over the finite timestep. In practice, the correction $g(\omega)$ is included in the colored noise generator. For the latter, two different implementations have been proposed in the context of standard QTB ${ }^{17,38}$. Although algorithmically different, these two methods are conceptually similar: they are based on a convolution between a white noise $r(t)$ and the function $H(t)$ derived from the square root of the power spectrum:

$$
F(t)=\int_{-\infty}^{+\infty} d \tau H(\tau) r(t-\tau)
$$

with

$$
H(t)=\frac{1}{2 \pi m} \int_{-\infty}^{+\infty} d \omega \mathrm{e}^{i \omega t} \sqrt{C_{F F}(\omega)}
$$

To implement the noise correction, the power spectrum $C_{F F}(\omega)$ is simply replaced by $C_{F F}(\omega) g(\omega)$.

\section{Integration of the generalized friction}

In the case of the adQTB-f method, the integration algorithm must be modified to account for the generalized friction force. To proceed, we first rewrite the generalized Langevin equation (22) as:

$$
m \dot{v}=-\frac{\partial V}{\partial x}-m \gamma v(t)-m \int_{0}^{\infty} \tilde{\gamma}_{f}^{\prime}(\tau) v(t-\tau) d \tau+F(t)
$$

In the expression above, we have split the friction force in two parts: a Markovian term with a coefficient $\gamma$ that remains constant along the trajectory, and a nonMarkovian term with a friction kernel $\tilde{\gamma}_{f}^{\prime}(\tau)$ that is adjusted on the fly during the dynamics to enforce the quantum FDT. To compute the convolution product in 'eq. (29), we use an extended-variable strategy ${ }^{16,45,46}$ and decompose $\gamma_{f}^{\prime}(\omega)$ as a sum of Lorentzian functions centered on discrete frequencies $\omega_{j}$ separated by $\Delta \omega$. This approach is convenient with respect to direct summation over the past time steps, due to the potentially slow decay of the non-Markovian memory kernel $\tilde{\gamma}_{f}^{\prime}(t)$. This kernel is written as:

$$
\tilde{\gamma}_{f}^{\prime}(\tau)=\frac{2 \Delta \omega}{\pi} \Theta(\tau) \sum_{j=0}^{n_{\omega}} \gamma_{j} \mathrm{e}^{-\alpha \tau} \cos \left(\omega_{j} \tau\right)
$$

Or, equivalently,

$$
\gamma_{f}^{\prime}(\omega)=\frac{\Delta \omega}{\pi} \sum_{j=0}^{n_{\omega}} \frac{\gamma_{j}}{\alpha+i\left(\omega-\omega_{j}\right)}+\frac{\gamma_{j}}{\alpha+i\left(\omega+\omega_{j}\right)}
$$

The Heaviside function $\Theta(\tau)$ ensures the causality of $\tilde{\gamma}_{f}^{\prime}$, and the normalizing constants are such that if all $\gamma_{j}$ are chosen equal to $\gamma_{0}$, then $\operatorname{Re}\left[\gamma_{f}^{\prime}(\omega)\right] \simeq \gamma_{0}$ is nearly independent of $\omega$ and $\tilde{\gamma}_{f}^{\prime}(\tau) \simeq \gamma_{0} \delta(\tau)$, which corresponds to a Markovian friction with rate $\gamma_{0}$. In equation (29), the non-Markovian term describes only the difference from the Markovian friction, therefore all coefficients $\gamma_{j}$ are initialized to zero (which corresponds to the standard QTB method), and only vary because of the adaptation procedure described in more details in the next section. The parameter $\alpha$ characterizes the width of the Lorentzian functions, in this work we chose $\alpha=\Delta \omega$ with a value smaller than $\gamma$, to ensure that eq. (31) provides a sufficiently accurate decomposition of the friction kernel. The number $n_{\omega}$ of Lorentzian terms is chosen such that $n_{\omega} \Delta \omega=\omega_{\text {cut }}$. The non-Markovian friction force is then rewritten as:

$$
\begin{aligned}
& -m \int_{0}^{\infty} \tilde{\gamma}_{f}^{\prime}(\tau) v(t-\tau) d \tau \\
& =-m \frac{2 \Delta \omega}{\pi} \sum_{j=0}^{n_{\omega}} \gamma_{j} \operatorname{Re}\left[\int_{0}^{\infty} \mathrm{e}^{\left(-\alpha+i \omega_{j}\right) \tau} v(t-\tau) d \tau\right] \\
& \equiv-m \frac{2 \Delta \omega}{\pi} \sum_{j=0}^{n_{\omega}} \gamma_{j} \operatorname{Re}\left[\tilde{v}_{j}(t)\right]
\end{aligned}
$$

In this expression, we have defined the (complex) auxiliary velocities $\tilde{v}_{j}(t)$, so that the integration of the generalized friction transforms into a simple sum over the 
auxiliary $\tilde{v}_{j}(t)$. Based on their definition, the complex velocities evolve according to:

$$
\frac{\partial}{\partial t} \tilde{v}_{j}(t)=\left(-\alpha+i \omega_{j}\right) \tilde{v}_{j}(t)+v(t)
$$

The rewriting of the non-Markovian term and the definition of the auxiliary variables enable the propagation of eq. (29) with an integration scheme still based on the BAOAB procedure described in section A 1 with two extra steps inserted just after step O. These two steps, denoted $(a)$ and $(b)$, are designated as $O^{\prime}$ in the flux diagram of Fig. 11. In step $(a), v(t)$ is propagated according to equation (32), while in step $(b)$, the auxiliary velocities are integrated as:

$$
\tilde{v}_{j}(t+\Delta t)=\mathrm{e}^{\left(-\alpha+i \omega_{j}\right) \Delta t} \tilde{v}_{j}(t)+v(t) \Delta t
$$

This integration procedure for the adQTB-f method was chosen for its relative simplicity of implementation, but it might still be optimized in future work in order to reduce the computational burden of the method.

In the above, we considered a one-dimensional system for simplicity. As mentioned in the text, in more general cases, we apply the same procedure for each degree of freedom of the system independently.

\section{B. ADAPTATION OF THE SYSTEM-BATH COUPLING COEFFICIENTS}

As outlined in the main text, the adaptation procedures are slightly different for the two flavours of the adQTB method. The main and common aim of these two strategies is to enforce the quantum fluctuationdissipation theorem for each degree of freedom $k$ and each frequency $\omega$ of the system:

$$
\Delta_{F D T, k}(\omega)=\operatorname{Re}\left[C_{v F, k}(\omega)\right]-m \gamma_{r, k}(\omega) C_{v v, k}(\omega)=0
$$

To this end, one needs a systematic way to evaluate $C_{v F, k}(\omega)$ and $C_{v v, k}(\omega)$ and to adjust either the noise kernel $\gamma_{r, k}(\omega)$ (in the adQTB-r method) or the dissipative kernel $\gamma_{f, k}(\omega)$ (in the adQTB-f method).

\section{Adapting the random noise $\gamma_{r, k}(\omega)$ with adQTB-r}

Within the adQTB-r strategy, the dissipative kernel $\gamma_{f, k}(\omega)=\gamma$ remains constant and frequencyindependent, corresponding to a Markovian friction force similar to that of the standard QTB. The frequencydependent noise kernel $\gamma_{r, k}(\omega)$ is introduced so that the autocorrelation function of the random force $F_{k}(t)$ is given by:

$$
C_{F F, k}(\omega)=2 m \gamma_{r, k}(\omega) \theta(\omega, T)
$$

For each degree of freedom $k$ of the system, the adaptation of the random force coefficient $\gamma_{r, k}(\omega)$ is carried out independently, starting from the initial value $\gamma_{r, k}(\omega)=\gamma$. To evaluate $\Delta_{F D T}$ during the simulation, the total trajectory is decomposed into a series of segments of duration $\tau$. These segments will be identified by the index $n$. At the end of each segment $n$, estimates of the correlation functions $\operatorname{Re}\left[\bar{C}_{v F, k}^{(n)}(\omega)\right]$ and $\bar{C}_{v v, k}^{(n)}(\omega)$ are evaluated through the Fourier transform of the noise and of the velocity:

$$
\begin{aligned}
\operatorname{Re}\left[\bar{C}_{v F, k}^{(n)}(\omega)\right] & =\frac{1}{2 \tau}\left(v_{k}^{(n)}[\omega] F_{k}^{(n)}[\omega]^{*}+v_{k}^{(n)}[\omega]^{*} F_{k}^{(n)}[\omega]\right) \\
\bar{C}_{v v, k}^{(n)}(\omega) & =\frac{1}{\tau}\left|v_{k}^{(n)}[\omega]\right|^{2}
\end{aligned}
$$

Where $v_{k}^{(n)}[\omega]$ and $F_{k}^{(n)}[\omega]$ are the Fourier transform of $v_{k}(t)$ and $F_{k}(t)$ respectively, taken over the $n$-th segment:

$$
\begin{aligned}
v_{k}^{(n)}[\omega] & =\int_{(n-1) \tau}^{n \tau} d t \mathrm{e}^{-i \omega t} v_{k}(t) \\
F_{k}^{(n)}[\omega] & =\int_{(n-1) \tau}^{n \tau} d t \mathrm{e}^{-i \omega t} F_{k}(t)
\end{aligned}
$$

The deviation from the fluctuation-dissipation theorem $\Delta_{F D T, k}$ over a given segment $n$ is then easily estimated by combining eq. (37) and (38). The corresponding estimate is denoted by $\bar{\Delta}_{F D T, k}^{(n)}(\omega)$. Different adjustment schemes can be used for $\gamma_{r, k}(\omega)$ in order to satisfy $\bar{\Delta}_{F D T, k}^{(n)}(\omega) \simeq 0$. In this work we follow a simple procedure. For all the relevant values of $\omega$, i.e. all the discrete values $\omega_{j}=\frac{2 \pi j}{\tau}$ inferior to the cutoff angular frequency $\omega_{c u t}$, the coefficients are slowly adjusted according to the following differential equation:

$$
\frac{d}{d t} \gamma_{r, k}\left(\omega_{j}\right)=A_{\gamma} \gamma \frac{\bar{\Delta}_{F D T, k}\left(\omega_{j}\right)}{\left\|\overline{\boldsymbol{\Delta}}_{\mathbf{F D T}, \mathbf{k}}\right\|}
$$

Where the norm of the deviation to the fluctuationdissipation theorem is defined as:

$$
\left\|\overline{\boldsymbol{\Delta}}_{\mathbf{F D T}, \mathbf{k}}\right\|=\sqrt{\sum_{\omega_{j}<\omega_{c u t}} \bar{\Delta}_{F D T, k}\left(\omega_{j}\right)^{2}}
$$

In practice, at the end of each segment, the value of the $\gamma_{r, k}$ coefficients is incremented according to:

$$
\gamma_{r, k}^{(n+1)}(\omega)=\gamma_{r, k}^{(n)}(\omega)+A_{\gamma} \gamma \tau \frac{\bar{\Delta}_{F D T, k}^{(n)}(\omega)}{\left\|\bar{\Delta}_{\mathbf{F D T}, \mathbf{k}}^{(\mathbf{n})}\right\|}
$$

The random force $F^{(n+1)}(t)$ used for the next segment is then generated using the kernel $\gamma_{r, k}^{(n+1)}(\omega)$ and the procedure is iterated until the $\gamma_{r, k}(\omega)$ are converged. When convergence is reached, the adQTB-r results are 

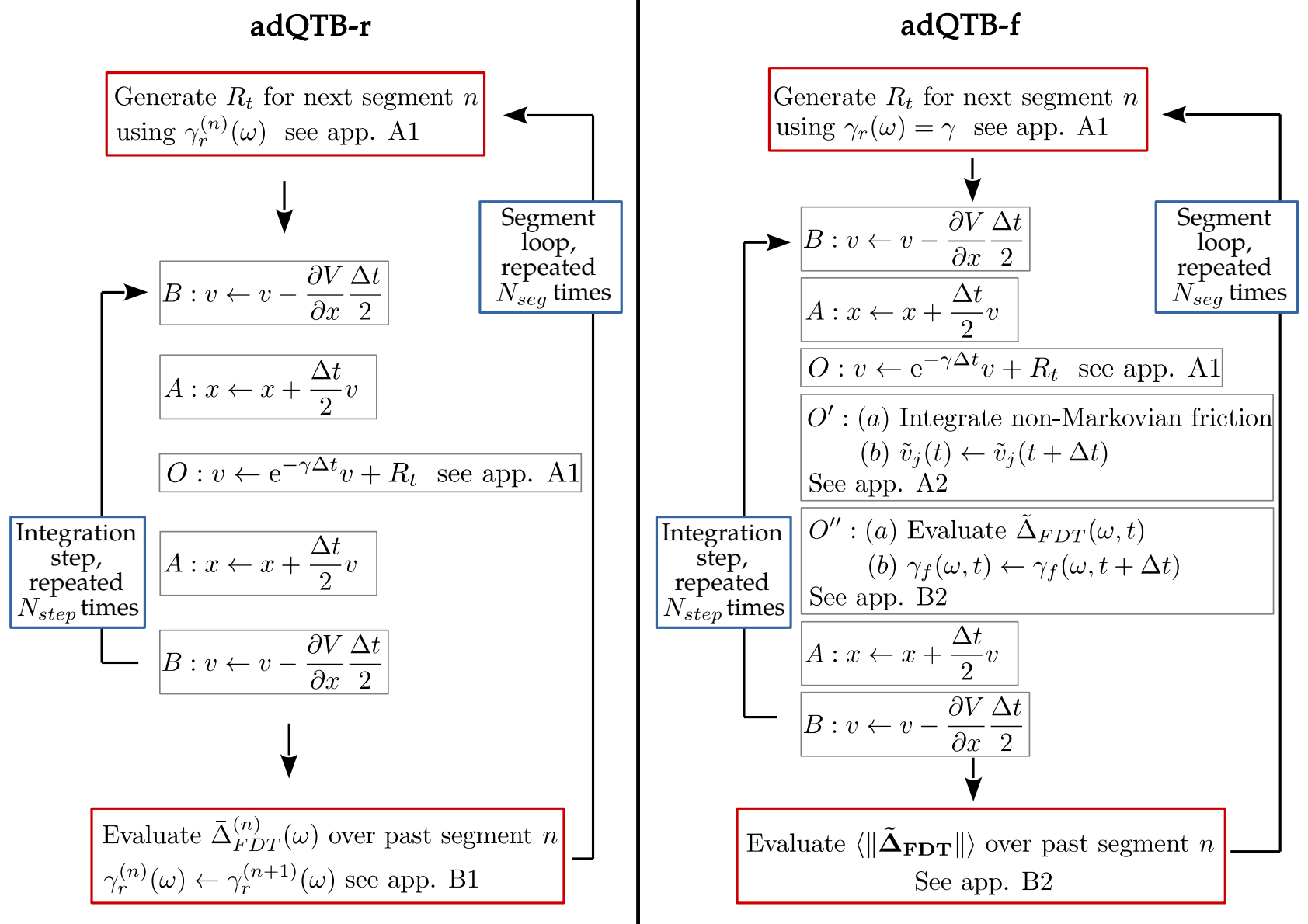

FIG. 11. Flux diagrams of the two adQTB algorithm. The figure describes the modifications introduced with respect to the standard BAOAB algorithm for the Langevin equation ${ }^{43,44}$. The adQTB trajectories are decomposed in a series of $N_{\text {seg }}$ segments of $N_{\text {step }}$ time steps each, the segments have a duration $\tau=N_{\text {step }} \Delta t$. One-dimensional notations are used for simplicity.

produced, while keeping the adaptation process active (which means that the $\gamma_{r, k}(\omega)$ continue to fluctuate slightly around their optimal value during the production).

The parameter $A_{\gamma}$ determines the adaptation velocity for the random force kernel. In all the examples presented in this article, we used small values of $A_{\gamma}$, such that the typical adaptation time for $\gamma_{r, k}(\omega)$ is much longer than $\tau$, and $\tau$ itself is chosen greater than $\gamma^{-1}$. Using large values for $A_{\gamma}$ might allow a faster convergence towards the desired energy distribution, as given by the quantum FDT, but it also increases the fluctuations of the coupling coefficients $\gamma_{r, k}^{(n)}(\omega)$. These increased fluctuations (in the sense of fluctuations as a function of $\omega$ as well as variations between the different segments $n$ ) can alter the accuracy of the adQTB method. However, it is worth noting that in large systems such as the aluminium crystal of section VA, the fluctuations on the FDT estimate $\bar{\Delta}_{F D T}(\omega)$ might be significantly reduced by averaging over equivalent degrees of freedom, which should allow for a substantial increase of the parameter $A_{\gamma}$. It might also be possible to speed-up the adaptation procedure using a second-order differential equation for the evolution of the $\gamma_{r}$ or other more elaborate convergence schemes. Finally, in cases in which a cheap approximation of the atom-atom interaction potential is available, it might be useful to perform a preliminary adaptation run using this approximation. This would allow to start the more accurate simulation with a partially adapted set of system-bath coefficients in order to reduce the adaptation time. A more detailed and systematic study of the effect of $A_{\gamma}$ on the convergence and accuracy of the adQTB technique will be performed in future work.

\section{Adapting the dissipative kernel $\gamma_{f, k}^{\prime}(\omega)$ with adQTB-f}

In the adQTB-f strategy, $\gamma_{r, k}(\omega)=\gamma$ remains constant and frequency-independent, while the frequencydependent friction kernel $\gamma_{f, k}^{\prime}(\omega)$, defined in eq. (29), is adjusted on the fly in order to enforce the quantum FDT. 
To propagate the non-Markovian friction force we use the auxiliary velocities $\tilde{v}_{j, k}(t)$ defined for each degree of freedom $k$ as in equation (32). In addition, we show in this section that the auxiliary velocities enable to evaluate the deviation from the FDT at each timestep. We first note that, in the limit of small $\alpha,\left|\tilde{v}_{j, k}(t)\right|^{2}$ provides an estimate of $C_{v v, k}\left(\omega_{j}\right)$, computed at time $t$ and integrated over a duration of the order of $\alpha^{-1}$ :

$$
\begin{aligned}
\left|\tilde{v}_{j, k}(t)\right|^{2} & =\frac{1}{2 \alpha} \int_{-\infty}^{\infty} d \tau \mathrm{e}^{-\alpha|\tau|-i \omega_{j} \tau} C_{v v, k}^{(\alpha)}(\tau, t) \\
\text { with } C_{v v, k}^{(\alpha)}(\tau, t) & =2 \alpha \int_{-\infty}^{t} d t^{\prime} \mathrm{e}^{-2 \alpha\left(t-t^{\prime}\right)} v_{k}\left(t^{\prime}\right) v_{k}\left(t^{\prime}-\tau\right)
\end{aligned}
$$

In order to estimate the velocity-force correlation function $C_{v F, k}\left(\omega_{j}\right)$, we define auxiliary forces in analogy with eq. (32) for the auxiliary velocities:

$$
\tilde{F}_{j, k}(t)=\int_{0}^{\infty} \mathrm{e}^{\left(-\alpha+i \omega_{j}\right) \tau} F_{k}(t-\tau) d \tau
$$

These auxiliary variables are propagated as the $\tilde{v}_{j, k}$ and they provide an estimate of $C_{v F, k}\left(\omega_{j}\right)$, computed at time $t$ over a duration of approximately $\alpha^{-1}$ :

$$
\begin{aligned}
\operatorname{Re}\left[\tilde{v}_{j, k}(t) \tilde{F}_{j, k}(t)^{*}\right] & =\frac{1}{2 \alpha} \int_{-\infty}^{\infty} d \tau \mathrm{e}^{-\alpha|\tau|-i \omega_{j} \tau} \operatorname{Re}\left[C_{v F, k}^{(\alpha)}(\tau, t)\right] \\
\text { with } C_{v F, k}^{(\alpha)}(\tau, t) & =2 \alpha \int_{-\infty}^{t} d t^{\prime} \mathrm{e}^{-2 \alpha\left(t-t^{\prime}\right)} v_{k}\left(t^{\prime}\right) F_{k}\left(t^{\prime}-\tau\right)
\end{aligned}
$$

These approximations for the correlation functions are valid in the limit of small $\alpha$ values, in practice, $\alpha$ should be chosen at least a few times smaller than $\gamma$ for the approximation to be satisfactory in a QTB simulation. This in turns imposes some constraints on the adaptation of the coefficients $\gamma_{j, k}$, since $\alpha^{-1}$ is the typical timescale for the variations of the auxiliary variables $\tilde{v}_{j, k}$ and $\tilde{F}_{j, k}$. Given these estimators, the correlation functions in eq. (35) can then be computed at time $t$ via the following relations:

$$
\begin{aligned}
\operatorname{Re}\left[\tilde{C}_{v F, k}\left(\omega_{j}, t\right)\right] & =\operatorname{Re}\left[\tilde{v}_{j, k}(t) \tilde{F}_{j, k}(t)^{*}\right] \\
\tilde{C}_{v v, k}\left(\omega_{j}, t\right) & =\left|\tilde{v}_{j, k}(t)^{2}\right|
\end{aligned}
$$

The deviation from the FDT at time $t$ and frequency $\omega_{j}$ is obtained combining eq. (45) and (46) and the corresponding estimate is denoted $\tilde{\Delta}_{F D T, k}\left(\omega_{j}, t\right)$. The friction coefficients are then adjusted according to a differential equation similar to the one introduced in the adQTB-r case:

$$
\frac{d}{d t} \gamma_{j, k}=-A_{\gamma} \gamma \frac{\tilde{\Delta}_{F D T, k}\left(\omega_{j}, t\right)}{\left\langle\left\|\tilde{\Delta}_{\mathbf{F D T}, \mathbf{k}}\right\|\right\rangle}
$$

In this equation, the norm $\left\|\tilde{\boldsymbol{\Delta}}_{\mathbf{F D T}, \mathbf{k}}\right\|$ of the deviation from the FDT is averaged over a duration $\tau$ similar to that used in the adQTB-r variant, this averaging has to be introduced in order to avoid biases due to the fast fluctuations of the instantaneous value $\tilde{\Delta}_{F D T, k}\left(\omega_{j}, t\right)$. Note that in the adQTB-f variant, the differential equation (47) is integrated and the values of the $\gamma_{j, k}$ modified at each time step of the dynamics, and not only at the end of each segment of trajectory as it is the case in the adQTB$\mathrm{r}$ strategy. The numerical overhead associated with the adQTB-f variant is therefore considerably higher than that of the adQTB-r. However, the propagation of the auxiliary variables and of the coefficients $\gamma_{j, k}$ does not require additional evaluations of the inter-atomic forces with respect to a standard QTB dynamics. Therefore the associated numerical overhead should remain unimportant in the case of $a b$ initio MD simulations, in which the calculation of the inter-atomic forces constitutes the main computational burden.

Finally, the remarks made in the previous section about the choice of the parameter $A_{\gamma}$ also apply to the adQTB-f case.

\section{Additional remark}

The following remark is valid for both adaptive methods (adQTB-r and adQTB-f) and regards the correlation function estimates $\tilde{C}_{v F, k}(\omega, t)$ and $\bar{C}_{v F, k}^{(n)}(\omega)$. For the sake of clarity, in this section, the degree of freedom index $k$ is dropped and the correlation function is simply denoted $C_{v F}(\omega)$.

Due to our choice of integrator (see appendix A 1), the numerical values for the random force $F(t)$ and the velocity $v(t)$ do not correspond to the exact same time $t$. Indeed, in the BAOAB scheme, the random force is applied in the middle of the time step (step O), whereas the velocity is saved at the end of each step, so that they are shifted by $\Delta t / 2$. Therefore, the procedures presented in the this appendix do not give access to $C_{v F}(\omega)$ directly but to an approximation of it, which will be denoted by $\mathcal{C}_{v F}(\omega)$. The discrepancy induced on the correlation spectrum can be significant, even comparable in amplitude with the ZPEL if the time step $\Delta t$ is large. To correct this discrepancy, we first use eq. (24) to write:

$$
\begin{aligned}
v\left(t-\frac{\Delta t}{2}\right) & =\mathrm{e}^{+\gamma \frac{\Delta t}{2}} v(t)-F(t) \frac{\Delta t}{2 m} \\
& =v(t)-\frac{\Delta t}{2}\left(\frac{F(t)}{m}-\gamma v(t)\right)+o(\Delta t)
\end{aligned}
$$

Then, a first-order Taylor expansion of $\mathcal{C}_{v F}(\omega)$ gives:

$$
\begin{aligned}
& \operatorname{Re}\left[\mathcal{C}_{v F}(\omega)\right] \\
& =\frac{1}{2} \int_{-\infty}^{+\infty} d t \mathrm{e}^{-i \omega \tau}\left\langle v\left(t-\frac{\Delta t}{2}\right) F(t-\tau)+F(t) v\left(t-\frac{\Delta t}{2}-\tau\right)\right\rangle \\
& =\operatorname{Re}\left[C_{v F}(\omega)\right]-\frac{\Delta t}{2}\left(\frac{C_{F F}(\omega)}{m}-\gamma \operatorname{Re}\left[C_{v F}(\omega)\right]\right)+o(\Delta t)
\end{aligned}
$$


Finally we find:

$$
\operatorname{Re}\left[C_{v F}(\omega)\right] \approx \frac{\operatorname{Re}\left[\mathcal{C}_{v F}(\omega)\right]+\frac{\Delta t}{2 m} C_{F F}(\omega)}{1+\gamma \frac{\Delta t}{2}}
$$

This corrected spectrum $C_{v F}(\omega)$ was used throughout this article for the evaluation of the deviation to the quantum fluctuation-dissipation theorem.

\section{LARGE SYSTEM-BATH COUPLING}

To illustrate the effects of the magnitude of $\gamma$ on QTB and adQTB simulations, we present in this appendix the results obtained for the $\mathrm{Ne}_{13}$ cluster with a large systembath coupling constant, namely $\gamma=4 \mathrm{THz}$. In order to analyze these results in more detail, we begin by recalling the effects of large $\gamma$ values in standard QTB simulations in the harmonic oscillator case. This case was examined in particular in Ref. 38 and 39.

\section{The harmonic oscillator case}

For a harmonic oscillator at the angular frequency $\omega_{0}$, the Langevin equations of motion are linear and the average kinetic and potential energies obtained with the QTB method are given by the following analytical expressions $^{38}$ :

$$
\begin{aligned}
\left\langle E_{\text {pot }}\left(\omega_{0}\right)\right\rangle & =\int_{0}^{\omega_{\text {cut }}} \frac{d \omega}{\pi} \frac{\gamma \omega_{0}^{2}}{\left(\omega^{2}-\omega_{0}^{2}\right)^{2}+\gamma^{2} \omega^{2}} \theta(\omega, T) \\
& \equiv \int_{0}^{\omega_{\text {cut }}} \frac{d \omega}{\pi} f_{\text {pot }}(\omega) \\
\left\langle E_{\text {kin }}\left(\omega_{0}\right)\right\rangle & =\int_{0}^{\omega_{\text {cut }}} \frac{d \omega}{\pi} \frac{\gamma \omega^{2}}{\left(\omega^{2}-\omega_{0}^{2}\right)^{2}+\gamma^{2} \omega^{2}} \theta(\omega, T) \\
& \equiv \int_{0}^{\omega_{\text {cut }}} \frac{d \omega}{\pi} f_{\text {kin }}(\omega)
\end{aligned}
$$

As noted in Ref. 38 , a cutting angular frequency $\omega_{\text {cut }}$ has to be introduced in the definition of the random force in order to avoid the divergence of the integral defining the kinetic energy (note that $\theta(\omega, T) \simeq \hbar \omega / 2$ when $\omega$ is large). For $\gamma \ll \omega_{0}$, the Lorentzian factor in the integrals can be approximated with a Dirac $\delta$-function and as a result $\left\langle E_{\text {pot }}\left(\omega_{0}\right)\right\rangle \simeq\left\langle E_{k i n}\left(\omega_{0}\right)\right\rangle \simeq \theta\left(\omega_{0}, T\right) / 2$, which corresponds to the exact quantum result. However, when $\gamma$ is comparable with $\omega_{0}$, the spectral broadening alters the QTB estimates for the average energies. This is illustrated in Fig. 12, with a choice of QTBparameters similar to the ones used for the simulations of the $\mathrm{Ne}_{13}$ cluster. Fig. 12.a presents the difference between the exact quantum result and the QTB-estimates for the average potential and kinetic energies for $\gamma=2$ and $4 \mathrm{THz}$. It shows that in the frequency range relevant for the $\mathrm{Ne}_{13}$ cluster (typically between $2 \times 10^{12}$ and $10 \times 10^{12} \mathrm{rad} / \mathrm{s}$ ), as shown in Fig. 9), the error in the
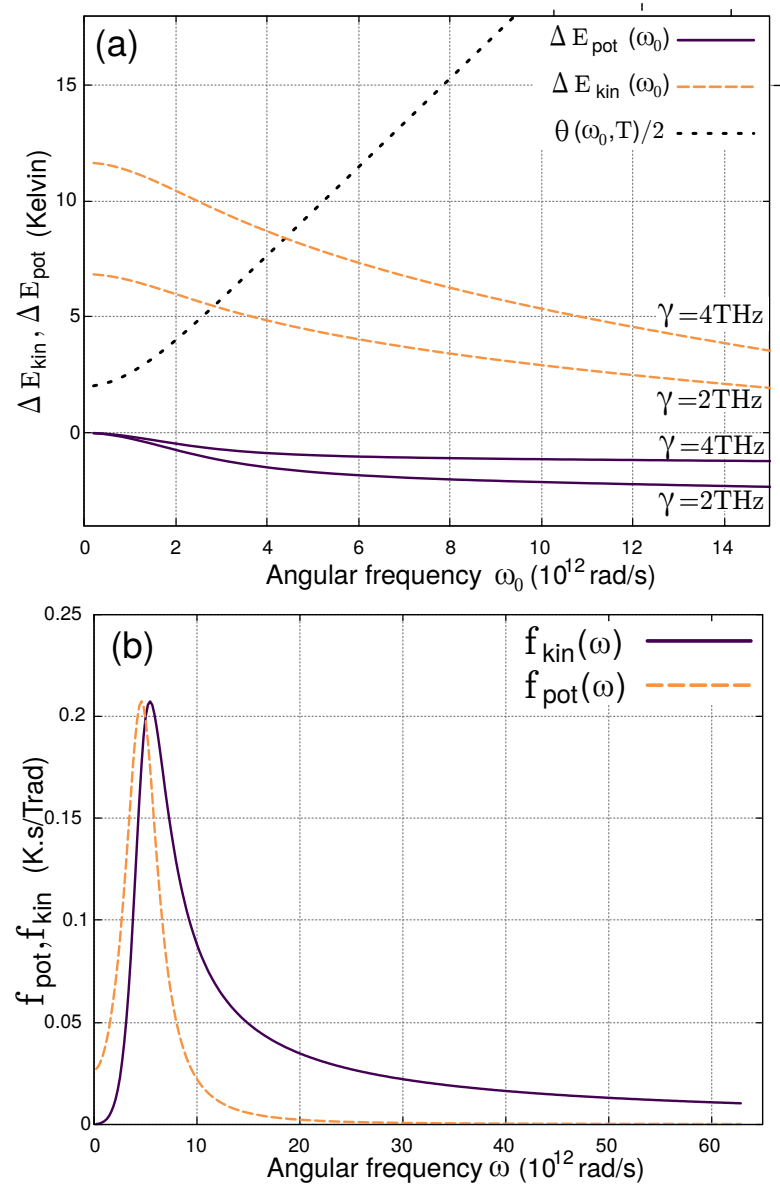

FIG. 12. Panel a: Difference $\Delta E$ between the QTB average kinetic and potential energy - as given by (54) and (55) - and the exact quantum result for a harmonic oscillator as a function of its angular frequency $\omega_{0}$. The temperature is $T=4 \mathrm{~K}$, and the cutting angular frequency is $\omega_{\text {cut }}=63 \times 10^{12} \mathrm{rad} / \mathrm{s}$. Panel b: integrand $f_{\text {pot }}(\omega)$ and $f_{\text {kin }}(\omega)$ of the equations (54) and (55) for a harmonic oscillator at frequency $\omega_{0}=5 \times 10^{12} \mathrm{rad} / \mathrm{s}$. All the energies are scaled by $k_{b}$ and given in Kelvin.

QTB-estimates of the energies is significant. For instance, at $\omega_{0}=5 \times 10^{12} \mathrm{rad} / \mathrm{s}, E_{\text {pot }} / k_{b}$ is underestimated by about $1 \mathrm{~K}(2 \mathrm{~K}$ for $\gamma=4 \mathrm{THz})$ while $E_{k i n} / k_{b}$ is overestimated by about $4 \mathrm{~K}(8 \mathrm{~K}$ for $\gamma=4 \mathrm{THz})$. This represents an important fraction of the exact quantum result $\theta\left(\omega_{0}, T\right) / 2 k_{b} \simeq 10 \mathrm{~K}$. As shown in Fig. 12.b the overestimation of the kinetic energy is mostly due to the high-frequency tail of the integrand in equation (55).

\section{2. $\mathrm{Ne}_{13}$ clusters}

Fig. 13 shows the Lindemann index curves obtained for the $\mathrm{Ne}_{13}$ cluster with $\gamma=4 \mathrm{THz}$. We first note that increasing $\gamma$ seems to systematically improve both the adaptive and the standard QTB results in comparison with Fig. 10 of the main text. The value of $\delta_{L}$ obtained 
with both adQTB variants now diplays a sharp increase, very similar to the one observed in PIMD, only slightly shifted to lower temperatures. Even in standard QTB, such an increase of $\delta_{L}$ is visible at very low temperature, which is characteristic of the solid-like state. At temperature $T=4 \mathrm{~K}$, the adQTB now yields a small value for $\delta_{L}$, indicating that the the spurious atomic diffusion events discussed in the main text are essentially suppressed. These unphysical events are still present in the standard QTB simulations at this temperature, as evidenced in the large value for $\delta_{L}$ and confirmed by the study of the $g(r)$ function (not shown).

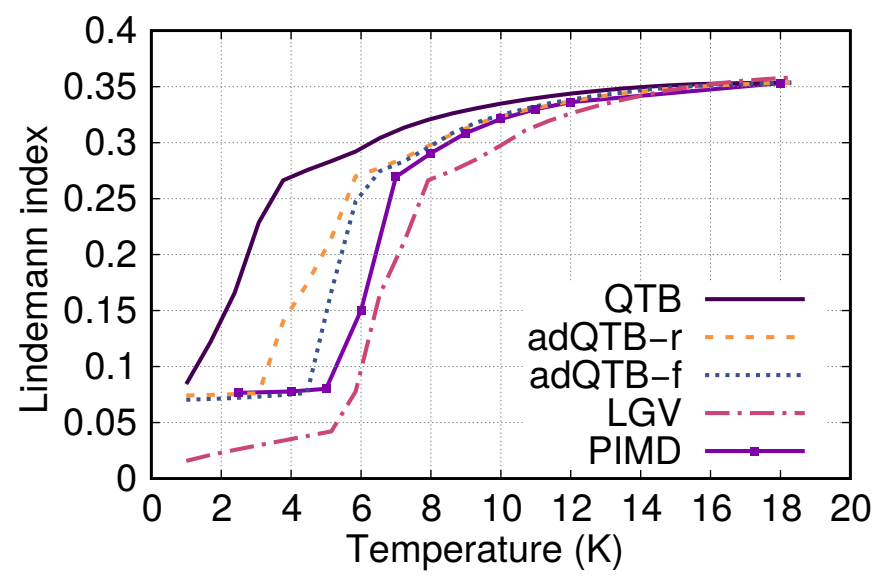

FIG. 13. Lindemann index $\delta_{L}$ from eq. (20), as a function of $\mathrm{T}$, for $\gamma=4 \mathrm{THz}$. The figure shows the results obtained with Langevin (LGV), standard QTB, adQTB-r and adQTB-f, and the reference results obtained from PIMD.

However, the improvement of the results for large $\gamma$ values should be taken with great caution since it might result from a fortunate cancellation of errors. Indeed, we showed in appendix $\mathrm{C} 1$, that for a harmonic oscillator with a frequency comparable to the typical vibration frequencies of the $\mathrm{Ne}_{13}$ cluster, the value $\gamma=4 \mathrm{THz}$ leads to sizable errors in the QTB simulations. The kinetic energy tends to be overestimated but since this excess of energy is mostly caused by high-frequency fluctuations of the velocity, it most probably has little impact on the structural properties of the cluster, as characterized by the pair correlation function $g(r)$. On the other hand, the potential energy tends to be increasingly underestimated when $\gamma$ increases. This leads to an underestimation of the zero-point motion of the atoms around their equilibrium position and hinders the atomic diffusion events in the $\mathrm{Ne}_{13}$ cluster. Therefore the better results obtained for $\gamma=4 \mathrm{THz}$ are probably due to the combination of two different effects: (i) the use of large $\gamma$ reduces the impact of the ZPEL and therefore tends to make the QTB (or adQTB) simulations more accurate, (ii) for large $\gamma$ values, the zero-point motion of the atoms is underestimated, therefore the diffusion events are spuriously suppressed.

This example illustrates the difficulties associated with the choice of $\gamma$ in QTB simulations of very anharmonic systems at low temperatures: on the one hand increasing $\gamma$ reduces the influence of the ZPEL and on the other hand it increases the spectral broadening of the modes and the associated errors. For that reason, the $\mathrm{Ne}_{13}$ cluster case is a particularly challenging one, that explores the limits of the QTB and ad QTB methods. However it should be stressed that the adQTB simulations consistently outperform the standard QTB, because they make it possible to control the leakage with smaller values of $\gamma$.

${ }^{1}$ R. P. Feynman, A. R. Hibbs, and D. F. Styer. Quantum mechanics and path integrals. Courier Corporation, 2010.

${ }^{2}$ D. Chandler and P. G. Wolynes. Exploiting the isomorphism between quantum theory and classical statistical mechanics of polyatomic fluids. J. Chem. Phys., 74(7):4078-4095, 1981.

${ }^{3}$ M. Parrinello and A. Rahman. Study of an F center in molten KCl. J. Chem. Phys., 80(2):860-867, 1984.

${ }^{4}$ D. M. Ceperley. Path integrals in the theory of condensed helium. Rev. Mod. Phys., 67:279-355, Apr 1995.

${ }^{5}$ I. R. Craig and D. E. Manolopoulos. Quantum statistics and classical mechanics: Real time correlation functions from ring polymer molecular dynamics. J. Chem. Phys., 121(8):3368-3373, 2004 .

${ }^{6}$ S. Habershon, D. E. Manolopoulos, T. E. Markland, and Miller T. F. Ring-polymer molecular dynamics: Quantum effects in chemical dynamics from classical trajectories in an extended phase space. Annu. Rev. Phys. Chem., 64:387-413, 2013.

${ }^{7}$ J. Cao and G. A. Voth. A new perspective on quantum time correlation functions. J. Chem. Phys., 99(12):10070-10073, 1993.

${ }^{8} \mathrm{~J}$. Cao and G. A. Voth. The formulation of quantum statistical mechanics based on the Feynman path centroid density. I. Equilibrium properties. J. Chem. Phys., 100(7):5093-5105, 1994.

${ }^{9}$ X. Sun, H. Wang, and W. H. Miller. Semiclassical theory of electronically nonadiabatic dynamics: Results of a linearized approximation to the initial value representation. J. Chem. Phys., 109:7064, 1998

${ }^{10}$ H. Wang, X. Sun, and W. H. Miller. Semiclassical approximations for the calculation of thermal rate constants for chemical reactions in complex molecular systems. J. Chem. Phys., 108:9726, 1998.

${ }^{11} \mathrm{Q}$. Shi and E. Geva. Semiclassical theory of vibrational energy relaxation in the condensed phase. J. Phys. Chem. A, 107:9059, 2003.

${ }^{12}$ J. A. Poulsen, G. Nyman, and P. J. Rossky. Static and dynamic quantum effects in molecular liquids: A linearized path integral description of water. PNAS, 102(19):6709-6714, 2005.

${ }^{13}$ M. Monteferrante, S. Bonella, and G. Ciccotti. Linearized symmetrized quantum time correlation functions calculation via phase pre-averaging. Mol. Phys., 109(23-24):3015-3027, 2011.

${ }^{14} \mathrm{~J}$. Beutier, D. Borgis, R. Vuilleumier, and S. Bonella. Computing thermal Wigner densities with the phase integration method. $J$. Chem. Phys., 141(8):084102, 2014.

${ }^{15}$ M. Ceriotti, G. Bussi, and M. Parrinello. Nuclear quantum effects in solids using a colored-noise thermostat. Phys. Rev. Lett., 103:030603, Jul 2009.

${ }^{16} \mathrm{M}$. Ceriotti, G. Bussi, and M. Parrinello. Colored-noise thermostats à la carte. J. Chem. Theory Comput., 6(4):1170-1180, 2010 .

${ }^{17}$ H. Dammak, Y. Chalopin, M. Laroche, M. Hayoun, and J.-J. Greffet. Quantum thermal bath for molecular dynamics simulation. Phys. Rev. Lett., 103(19):190601, November 2009.

${ }^{18}$ M. Ceriotti, G. Miceli, A. Pietropaolo, D. Colognesi, A. Nale, M. Catti, M. Bernasconi, and M. Parrinello. Nuclear quantum effects in ab initio dynamics: Theory and experiments for lithium imide. Phys. Rev. B, 82:174306, Nov 2010.

${ }^{19}$ Y. Chalopin, H. Dammak, M. Laroche, M. Hayoun, and J.-J. Greffet. Radiative heat transfer from a black body to dielectric nanoparticles. Phys. Rev. B, 84:224301, Dec 2011. 
${ }^{20} \mathrm{~T}$. Qi and E. J. Reed. Simulations of shocked methane including self-consistent semiclassical quantum nuclear effects. J. Phys. Chem. A, 116(42):10451-10459, 2012.

${ }^{21}$ H. Dammak, E. Antoshchenkova, M. Hayoun, and F. Finocchi. Isotope effects in lithium hydride and lithium deuteride crystals by molecular dynamics simulations. J. Phys. Condens. Matter, 24(43):435402, 2012.

${ }^{22}$ Y. Bronstein, P. Depondt, and F. Finocchi. Thermal and nuclear quantum effects in the hydrogen bond dynamical symmetrization phase transition of $\delta$-AlOOH. Eur. J. Mineral., 29(3):385-395, 2017.

${ }^{23}$ K. Drużbicki, M. Krzystyniak, D. Hollas, V. Kapil, P. Slavíček, G. Romanelli, and F. Fernandez-Alonso. Hydrogen dynamics in solid formic acid: insights from simulations with quantum colored-noise thermostats. J. Phys. Conf. Ser, 1055(1):012003, 2018.

${ }^{24}$ Y. Bronstein, P. Depondt, F. Finocchi, and A. M. Saitta. Quantum-driven phase transition in ice described via an efficient langevin approach. Phys. Rev. B, 89:214101, Jun 2014.

${ }^{25}$ Y. Bronstein, P. Depondt, L. E. Bove, R. Gaal, A. M. Saitta, and F. Finocchi. Quantum versus classical protons in pure and salty ice under pressure. Phys. Rev. B, 93:024104, Jan 2016.

${ }^{26}$ F. Calvo, N.-T. Van-Oanh, P. Parneix, and C. Falvo. Vibrational spectra of polyatomic molecules assisted by quantum thermal baths. Phys. Chem. Chem. Phys., 14(30):10503-10506, 2012.

${ }^{27}$ M. Rossi, V. Kapil, and M. Ceriotti. Fine tuning classical and quantum molecular dynamics using a generalized Langevin equation. J. Chem. Phys., 148(10):102301, 2018.

${ }^{28}$ J. M. Bowman, B. Gazdy, and Q. Sun. A method to constrain vibrational energy in quasiclassical trajectory calculations. $J$. Chem. Phys., 91(5):2859-2862, 1989.

${ }^{29}$ W. H. Miller, W. L. Hase, and C. L. Darling. A simple model for correcting the zero point energy problem in classical trajectory simulations of polyatomic molecules. J. Chem. Phys., 91(5):2863-2868, 1989

${ }^{30} \mathrm{~S}$. Habershon and D. E. Manolopoulos. Zero point energy leakage in condensed phase dynamics: An assessment of quantum simulation methods for liquid water. J. Chem. Phys., 131(24):244518, 2009.

${ }^{31}$ O. N. Bedoya-Martínez, J.-L. Barrat, and D. Rodney. Computation of the thermal conductivity using methods based on classical and quantum molecular dynamics. Phys. Rev. B, 89:014303, Jan 2014.

${ }^{32}$ F. Brieuc, Y. Bronstein, H. Dammak, P. Depondt, F. Finocchi, and M. Hayoun. Zero-point energy leakage in quantum thermal bath molecular dynamics simulations. J. Chem. Theory Com- put., 12:5688-5697, 2016

${ }^{33}$ Javier Hernández-Rojas, Florent Calvo, and Eva Gonzalez Noya. Applicability of quantum thermal baths to complex many-body systems with various degrees of anharmonicity. J. Chem. Theory Comput., 11(3):861-870, March 2015.

${ }^{34} \mathrm{M}$. Ceriotti, D. E. Manolopoulos, and M. Parrinello. Accelerating the convergence of path integral dynamics with a generalized Langevin equation. J. Chem. Phys., 134(8):084104, 2011.

${ }^{35}$ F. Brieuc, H. Dammak, and M. Hayoun. Quantum thermal bath for path integral molecular dynamics simulation. J. Chem. Theory Comput., 12(3):1351-1359, 2016. PMID: 26799437.

${ }^{36}$ C. Schran, F. Brieuc, and D. Marx. Converged colored noise path integral molecular dynamics study of the Zundel cation down to ultralow temperatures at coupled cluster accuracy. J. Chem. Theory Comput., 14(10):5068-5078, 2018.

${ }^{37}$ M. Buchholz, E. Fallacara, F. Gottwald, M. Ceotto, F. Grossmann, and S. D. Ivanov. Herman-Kluk propagator is free from zero-point energy leakage. Chem. Phys., 515:231 - 235, 2018.

${ }^{38}$ J.-L. Barrat and D. Rodney. Portable implementation of a quantum thermal bath for molecular dynamics simulations. J. Stat. Phys., 144(3):679-689, August 2011.

${ }^{39}$ M. Basire, D. Borgis, and R. Vuilleumier. Computing Wigner distributions and time correlation functions using the quantum thermal bath method: application to proton transfer spectroscopy. Phys. Chem. Chem. Phys., 15:12591-12601, 2013.

${ }^{40}$ R. Kubo. The fluctuation-dissipation theorem. Rep. Prog. Phys., 29(1):255, 1966.

${ }^{41}$ R. S. Berry, T. L. Beck, H. L. Davis, and J. Jellinek. Solid-Liquid Phase Behavior in Microclusters, pages 75-138. John Wiley \& Sons, Ltd, 2007.

${ }^{42} \mathrm{~J}$. Liu and W. H. Miller. Using the thermal Gaussian approximation for the Boltzmann operator in semiclassical initial value time correlation functions. J.Chem. Phys., 125(22):224104, 2006.

${ }^{43} \mathrm{~B}$. Leimkuhler and C. Matthews. Rational construction of stochastic numerical methods for molecular sampling. Appl. Math. Res. eXpress, June 2012.

${ }^{44}$ B. Leimkuhler and C. Matthews. Robust and efficient configurational molecular sampling via Langevin dynamics. J. Chem. Phys., 138(17):174102, 2013.

${ }^{45} \mathrm{~L}$. Kantorovich. Generalized langevin equation for solids. I. Rigorous derivation and main properties. Phys. Rev. B, 78(9):094304, 2008.

${ }^{46}$ A. D. Baczewski and S. D. Bond. Numerical integration of the extended variable generalized Langevin equation with a positive Prony representable memory kernel. J. Chem. Phys., 139(4):044107, 2013. 